\title{
Coupled Cell Model of Border Zone Arrhythmias*
}

\author{
Bradford E. Peercy ${ }^{\dagger}$ and James P. Keener ${ }^{\ddagger}$
}

\begin{abstract}
Border zones between normal and ischemic tissue have been implicated as a cause of arrhythmic cardiac activity. A variety of experiments with coupled cells and strips of tissue has been designed to understand the arrhythmogenic effects of border zone currents.

In this paper, we use systems of differential equations to model an ischemic (depolarized) cell (or region) coupled to a normal cell under a variety of conditions. For two ionic models (reduced Hodgkin-Huxley and Luo-Rudy I), we find the boundary in parameter space between oscillatory and nonoscillatory solutions. We find that there are regions in parameter space for which the ischemic cell (region) is stable and inexcitable when uncoupled, but when coupled to a normal, excitable cell the cells oscillate. We state a general principle that relates the oscillation of a forced single cell to oscillations of the coupled system. Furthermore, in modeling drug-modified experimental dynamics we are able to reproduce early after depolarization (EAD)-like phenomena, which has implications in locating oscillations in the drug-free experiment. Finally, we describe a mechanism by which oscillations in the transmembrane potential may be encountered during reperfusion.
\end{abstract}

Key words. AUTHOR: PLEASE PROVIDE

AMS subject classifications. AUTHOR: PLEASE PROVIDE

DOI. $10.1137 / 040615973$

1. Introduction. Cardiac tissue homeostasis is maintained through a complex network of coronary arteries distributed throughout the heart. Occlusion or blockage of a branch within this arterial network causes downstream blood flow to cease. The deprivation of blood flow to a region of tissue results in the loss of nutrients, such as glucose and oxygen, as well as the accumulation of waste products. This combination of effects is termed ischemia. With the loss of homeostasis due to ischemia, a cascade of biochemical processes occurs in the ischemic myocardium. Many of these processes negatively affect the electrical conduction system, which the heart uses to organize rhythmic pumping.

The size of the region of myocardium subjected to ischemic insult depends upon how distal the occlusion is in the branching network of coronary arteries from the origin of the network. If the occlusion occurs proximal to the origin of the network, prior to significant branching, a large portion of heart muscle is affected upon occlusion. On the other hand, if the blockage is much farther down the arterial tree, say, near the capillary level, possibly only a few cells are affected.

The central area of an ischemic region is the most affected. Tissue in the periphery of an ischemic region receives some level of collateral blood flow from the surrounding normally

\footnotetext{
${ }^{*}$ Received by the editors September 29, 2004; accepted for publication (in revised form) by M. Golubitsky December 8, 2004; published electronically DATE. This research was supported in part by NSF-DMS 0139926. http://www.siam.org/journals/siads/x-x/61597.html

${ }^{\dagger}$ Rice University, CAAM, 6100 Main Street, MS 134, Houston, TX 77005 (bpeercy@rice.edu).

${ }^{\ddagger}$ Department of Mathematics, University of Utah, 155 South 1400 East, Room 233, Salt Lake City, UT 84112 (keener@math.utah.edu).
} 
perfused myocardium. This periphery between highly ischemic and normal tissue was first identified by Harris and Rojas [10] as the "border zone." The portion of the border zone on the surface of the heart is the epicardial border zone, but the border zone extends endocardially into ventricular tissue through the midmyocardium and even to the endocardium.

It is well established $[11,3,4]$ that an important biochemical effect of ischemia is the increase of extracellular potassium $\left(\mathrm{K}_{0}^{+}\right) . \mathrm{K}_{0}^{+}$rises in a triphasic manner [4] and leads to elevated transmembrane potentials. Additionally, coupling between cells via gap junctions begins to decrease with the rise of intercellular proteins [2] such as lysophosphatidylcholine [6] and long chain acylcarnitine [23] as well as with the increase of both intercellular and extracellular $\mathrm{pH}[5]$. While the ischemic region is coupled to the normal tissue with normal resting membrane potential (RMP), there is a difference in transmembrane potentials across gap junctions. This difference induces an "injury current" across the border between ischemic and nonischemic tissue. The injury current exists throughout the border zone where there is a gradient of injury. Since cardiac tissue, as an excitable medium, can be forced by external currents, an injury current may be sufficient to create ectopic activity originating at the border zone.

High extracellular potassium and uncoupling of gap junctions in a given mass of tissue are important manifestations of acute cardiac ischemia. Tan and Joyner [21] developed a technique that coupled a single cardiac cell with variable resistance to a computer model cell. This design allowed the administration of chemicals to the cardiac cell and/or the mathematical adjustment of the model cell. Variable resistance between the cardiac cell and the model cell was interpreted as intercellular gap junction communication strength.

The following describes experiments designed in the framework mentioned above to study the effects of individual and combined aspects of ischemia on coupled cells. Tan, Osaka, and Joyner [22] coupled a real ventricular cell (VC) to a passive model cell with a depolarized RMP $(-20 \mathrm{mV},-10 \mathrm{mV}, 0 \mathrm{mV})$. The depolarized RMP mimicked the effect of increased extracellular potassium on a cardiac cell. The coupling conductance was varied (0nS, 3nS, $5 \mathrm{nS})$. No spontaneous repetitive change in transmembrane potential (also termed automaticity) was observed. Kumar and Joyner [13] later reconfirmed this result with a depolarized model cell RMP of $0 \mathrm{mV}$ coupled to a VC but noted that, with the application of certain drugs, isoproterenol (synthetic catecholamine which acts to stimulate $\beta_{1}$ and $\beta_{2}$ adrenergic receptors), froskolin and 8-bromo-cyclic adenosine monophosphate (which act to raise cAMP levels), and Bay K 8644 (slow $\mathrm{Ca}^{2+}$ channel agonist) to the VC, early after depolarizations (EADs) were observed. EADs could be produced within the $\mathrm{VC}$ while also being connected to the depolarized model cell only in the presence of these drugs. The change of the VC with these drugs, which primarily affect the calcium handling, altered the dynamics to allow for automaticity induced by the injury current.

Picard et al. [18, 20] performed experiments on strips of guinea pig cardiac tissue extending Rouet et al.'s work from 1989 [19]. Their goal was to find pharmaceutical remedies for automaticity at the ischemic border zone by affecting the $\mathrm{K}_{A T P}$ channel. They used a two-chamber setup where one chamber was perfused with an ischemic Tyrode's solution containing high $\mathrm{K}_{0}^{+}$, low $\mathrm{pH}$, no glucose, and a decreased partial pressure of oxygen, $\mathrm{pO}_{2}$. Their control experiments found spontaneous electrical behavior about one fourth of the time. After perfusion of half of the tissue preparation with the ischemic solution for 30 minutes, that part 
of the tissue was reperfused with a normal solution. In the control, $92 \%$ of the preparations exhibited spontaneous activity upon reperfusion.

These experiments attempt to understand the influence ischemic tissue exerts on normal tissue. The coupled cell experiments focus on the importance of depolarized resting transmembrane potential in ischemic tissue and the size of the ischemic region in generating automaticity. The strip of tissue experiments also emphasize the importance of elevated transmembrane potential in generating oscillations when coupled to normal tissue. However, these experiments fall short of providing a ubiquitous mechanism for automaticity, and previous modeling does not reproduce the automaticity observed in experiments.

The goal of this work is to understand how the depolarization of the ischemic myocardium coupled to normal myocardium during early ischemia generates spontaneous electrical activity independent of the normal automaticity of the heart. This is the generation of a so-called border zone arrhythmia. To this end, we develop and study a coupled cell model with two forms of ionics. In a subsequent paper we will provide an analysis of a one-dimensional spatial model. These models are inspired by the above-mentioned experiments.

1.1. Outline. In section 2 we derive a model of coupled cells where "cell" may be interpreted as a single cell or a region of isopotential cells. The degree of ischemia is described by the parameter vector, $\mathbf{p}$. One cell, taken to be a normal cell, has no ischemia, $\mathbf{p}_{0}$, whereas the other cell, taken to be the ischemic cell, has a variable degree of ischemia. A coupling parameter is associated with gap junctional proteins, which span both cell membranes linking them together electrically. The relative sizes of the normal and ischemic cells are also taken into account. This coupled system of ordinary differential equations is studied with two different models of transmembrane ionic currents, a reduced Hodgkin-Huxley (RHH) model, and a Luo-Rudy I (LRI) model.

In section 3, we describe the effect of increased ischemia on an individual cell and the effect of coupling an ischemic and normal cell of various relative sizes and coupling strengths. We alter the RHH model to account for changes due to ischemia by allowing for changes in $\mathrm{K}_{0}^{+}$. In the phase plane, the nullcline of the transmembrane potential, $V$, undergoes a characteristic shift in response to the change in $\mathrm{K}_{0}^{+}$. The LRI model is an eight variable ionic model with explicit dependence on $\mathrm{K}_{0}^{+}$, which we utilize.

Using steady state and bifurcation diagrams (state variable(s) versus parameter) and unfolding diagrams (parameter versus parameter), we show that there are spontaneous oscillations for parameters in an open region of parameter space. The oscillations observed in the LRI model are calcium activated. We briefly discuss why this, rather than sodium activation, is the case. From the analysis of spontaneous oscillations in both of the ionic models, a general principle emerges: how a single cell responds to constant stimulus and constant leak relates to oscillatory behavior of coupled cells in certain parameter ranges. We compare the results to the coupled cell experiments $[21,22,13,14]$ and give an explanation of why oscillations are not observed in some experiments $[22,13]$. We conclude that two cells, which when uncoupled are each stable, one excitable and the other inexcitable with elevated resting potential, can exhibit oscillations via an injury current when coupled together.

2. Model derivation. The models of cardiac cell ionics described here are based on the Hodgkin-Huxley formalism. The membrane of a cardiac cell is a bilipid layer that acts as a 


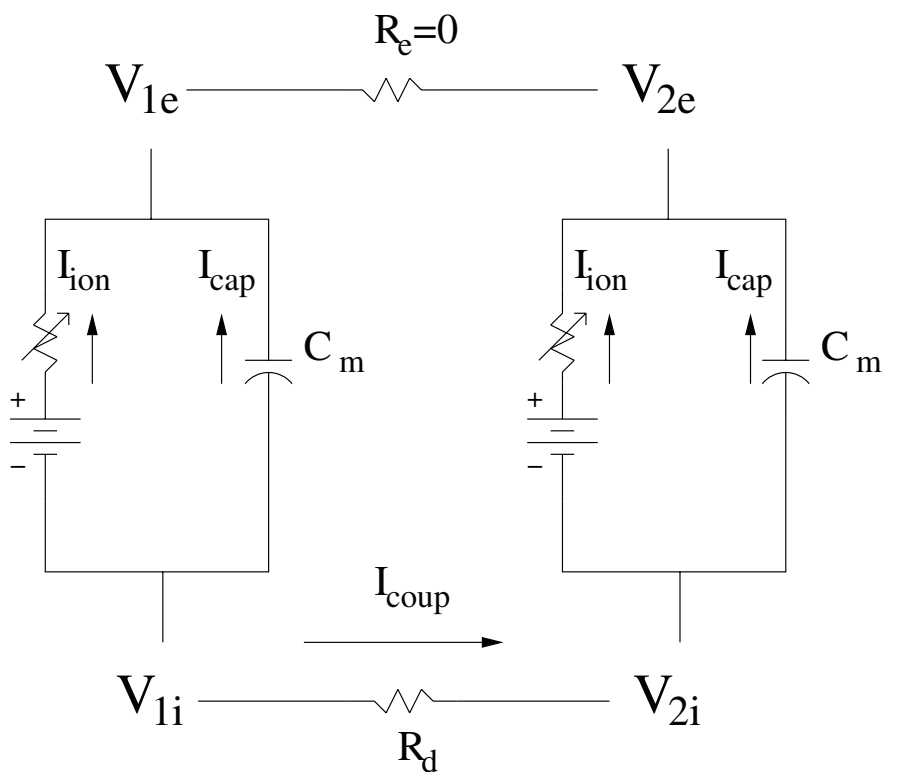

Figure 1. A schematic of two cells coupled intracellularly by the coupling current, $I_{\text {coup }}$. The ionic current, $I_{i o n}$, and capacitative current, $I_{\text {cap }}$, are balanced by the coupling current. $R_{d}$ is the coupling resistivity. $C_{m}$ is the membrane capacitance. $V_{1_{i}}$ and $V_{1_{e}}$ are the intracellular and extracellular potentials for region 1 , respectively, as are $V_{2_{i}}$ and $V_{2_{e}}$ for region 2. The extracellular potentials are taken to be isopotential $\left(V_{1_{e}}=V_{2_{e}}\right)$.

capacitor. Through this layer, penetrating proteins act as ion-specific conductors. Figure 1 provides a schematic representation of two excitable cells coupled through an intercellular resistance.

The total transmembrane current consisting of the ionic currents and the induced capacitive current must balance the current through the intercellular resistor, $I_{\text {coup }}$. The membrane current density is

$$
I_{m}=C_{m} \frac{d V_{1}}{d t}+I_{i o n}
$$

where $V_{1}=V_{1_{i}}-V_{1_{e}}, C_{m}$ is capacitance per unit area of membrane, and $I_{i o n}$ is the ionic current density. Using Ohm's law the intercellular current density is written in terms of the difference in intercellular potentials and the conductance per area, $d$, between them,

$$
I_{\text {coup }}=d\left(V_{1_{i}}-V_{2_{i}}\right),
$$

where $d$ is also the inverse intercellular resistivity, $R_{d}$. The conductance, $d$, is associated with the gap junctional conductance. For the currents induced from the flow of ions to balance, the surface areas across which the various currents flow must be taken into account. If $M_{1}$ represents the membrane surface area of cell 1 and $A_{i}$ represents the gap junctional surface area between cell 1 and cell 2, then the balance of currents for cell 1 is

$$
M_{1}\left(C_{m} \frac{d V_{1}}{d t}+I_{\text {ion }}\right)=-A_{i} I_{\text {coup }}=A_{i} d\left(V_{2_{i}}-V_{1_{i}}\right)
$$


and that for cell 2 is

$$
M_{2}\left(C_{m} \frac{d V_{2}}{d t}+I_{\text {ion }}\right)=A_{i} I_{\text {coup }}=A_{i} d\left(V_{1_{i}}-V_{2_{i}}\right)
$$

We assume that the extracellular potential is isopotential (i.e., $V_{1_{e}}=V_{2_{e}}$ ) so that the two coupled transmembrane potential equations become

$$
\begin{aligned}
& M_{1}\left(C_{m} \frac{d V_{1}}{d t}+I_{\text {ion }}\right)=A_{i} d\left(V_{2}-V_{1}\right), \\
& M_{2}\left(C_{m} \frac{d V_{2}}{d t}+I_{\text {ion }}\right)=A_{i} d\left(V_{1}-V_{2}\right) .
\end{aligned}
$$

2.1. Scaling parameters. It is useful to introduce scaled parameters in (2.1). Let $M_{0}$ be the total membrane surface area of the system (i.e., $M_{1}+M_{2}=M_{0}$ ), and let $m$ be the relative surface area of cell $1, m=\frac{M_{1}}{M_{0}}$, which is a nondimensional quantity. Notice that $1-m=\frac{M_{2}}{M_{0}}$. Then (2.1) takes the form

$$
\begin{aligned}
m\left(C_{m} \frac{d V_{1}}{d t}+I_{i o n}\right) & =\chi d\left(V_{2}-V_{1}\right), \\
(1-m)\left(C_{m} \frac{d V_{2}}{d t}+I_{i o n}\right) & =\chi d\left(V_{1}-V_{2}\right),
\end{aligned}
$$

where $\chi=\frac{A_{i}}{M_{0}}$ is ratio of the gap junctional area to total membrane surface area. The ionic current, $I_{\text {ion }}$, has many possible representations depending on what physiological model is being considered. A discussion of several ionic models can be found in Keener and Sneyd [12]. In general, it is assumed that the ionic current has a functional nonlinear dependence on the transmembrane potential, on gating variables and other state variables, and on state dependent parameters. For example,

$$
I_{\text {ion }}=-F(V, \mathbf{w}, \mathbf{p}) / R_{m},
$$

where $R_{m}$ is the passive membrane resistivity, $F$ has units of voltage, w represents the vector of gating variables and other state variables, and $\mathbf{p}$ represents a vector of parameters (i.e., $\mathrm{K}_{0}^{+}$, $\mathrm{pH}, \mathrm{ATP}$, etc.) which undergo ischemia-induced biochemical changes. Incorporating this into (2.2) yields

$$
\begin{gathered}
m\left(C_{m} R_{m} \frac{d V_{1}}{d t}-F\left(V, \mathbf{w}_{1}, \mathbf{p}_{0}\right)\right)=\chi R_{m} d\left(V_{2}-V_{1}\right), \\
(1-m)\left(C_{m} R_{m} \frac{d V_{2}}{d t}-F\left(V, \mathbf{w}_{2}, \mathbf{p}\right)\right)=\chi R_{m} d\left(V_{1}-V_{2}\right) .
\end{gathered}
$$

Notice that $C_{m} R_{m}$ has units of time and $R_{m} d$ is a nondimensional product of intercellular conductance per area and passive membrane resistivity, so that scaling time by $C_{m} R_{m}$ and 
letting $\delta=R_{m} d$ yield the model

$$
\begin{aligned}
m\left(\frac{d V_{1}}{d t}-F\left(V, \mathbf{w}_{1}, \mathbf{p}_{0}\right)\right) & =\chi \delta\left(V_{2}-V_{1}\right), \\
(1-m)\left(\frac{d V_{2}}{d t}-F\left(V, \mathbf{w}_{2}, \mathbf{p}\right)\right) & =\chi \delta\left(V_{1}-V_{2}\right) .
\end{aligned}
$$

The dynamics of gating variables for each of the cells are described by equations of the form

$$
\begin{aligned}
& \frac{d \mathbf{w}_{1}}{d t}=g\left(V_{1}, \mathbf{w}_{1}\right), \\
& \frac{d \mathbf{w}_{2}}{d t}=g\left(V_{2}, \mathbf{w}_{2}\right) .
\end{aligned}
$$

To model the effect of ischemia on the electrical interaction between cells, the degree of ischemia parameters, $\mathbf{p}$, are modified in one of the cells. For example, if $\mathbf{p}=p$, a scalar, represents $\mathrm{K}_{0}^{+}$, we let $p_{0}$ be $K_{0}^{+}$for a normal cell, while $p$ is $K_{0}^{+}$for an ischemic cell with $p>p_{0}$. Our goal is to understand the behavior of the above system as it depends on the parameters $m, p$, and $\chi \delta$.

3. Model ionics. We study variation in these parameters using two forms of ionics. The RHH model has a biophysical interpretation but is a two state variable model which is readily studied in the phase plane. The LRI model provides a more biophysically realistic model. We describe the effect of an increase in the degree of ischemia on an individual cell followed by bifurcation analysis of the coupled system in relative cell size and coupling strength for each set of ionics.

3.1. Reduced Hodgkin-Huxley model. The reduced Hodgkin-Huxley (RHH) ionic model is a basic ionic model that includes sodium and potassium ion currents. This model is found as a reduction of the four variable Hodgkin-Huxley model by assuming the sodium activation gate is fast and the potassium activation gate and sodium inactivation gate are linearly related. The two variable RHH model allows for phase plane analysis while maintaining an ionic interpretation. The fast-slow reduction of the full four variable Hodgkin-Huxley system is attributed to FitzHugh [8, 9]. A more recent discussion of this reduction may be found in [12]. The dynamics associated with the RHH system in the notation of the previous section are

$$
\begin{gathered}
F(V, n, p)=-\left[\bar{g}_{N a} m_{\infty}^{3}(0.85-n)\left(V-V_{N a}\right)+\bar{g}_{K} n^{4}\left(V-V_{K}(p)\right)+g_{L}\left(V-V_{L}\right)\right], \\
g(V, n)=\frac{n_{\infty}(V)-n}{\tau_{n}(V)},
\end{gathered}
$$

where the parameters are specified in Appendix A (see Keener and Sneyd [12]). The potassium Nernst potential, $V_{K}=25.8 \ln \left(\frac{p}{K_{i}}\right)$, depends explicitly on the parameter $p$, which here is extracellular potassium. In normal conditions for the RHH model $p=p_{0}=20$.

In the following section we show that an increase in extracellular potassium on the single RHH cell results in a shift from stable to self-oscillatory dynamics followed by a return to a stable but inexcitable, higher rest potential state. We then examine the coupled system and determine the effects of relative size and coupling strength between a normal RHH cell and an ischemic RHH cell. 


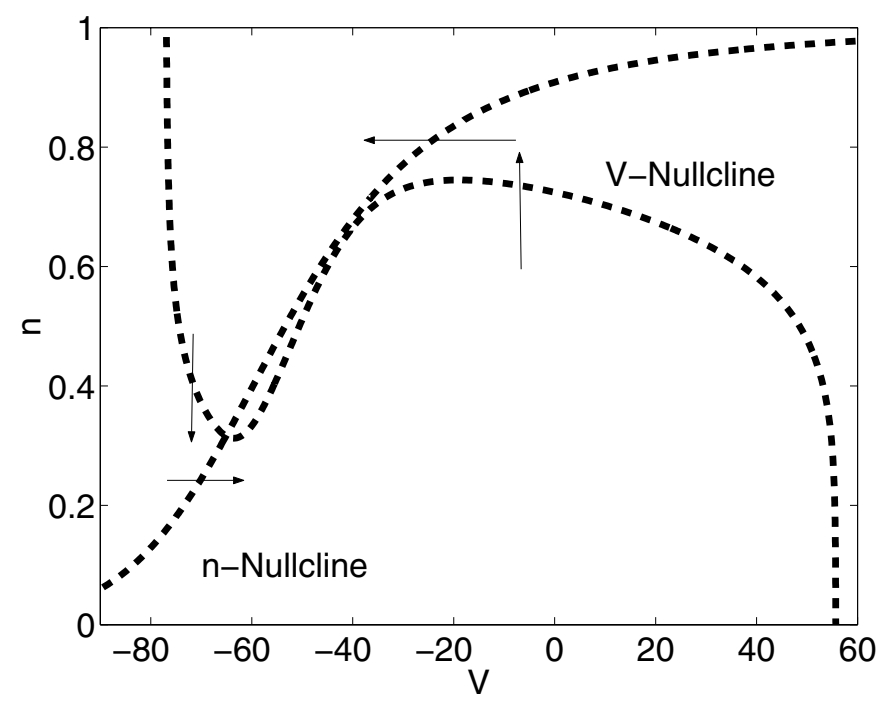

Figure 2. Phase plane diagram for the RHH model. The steady state is stable with extracellular potassium at a normal level, $p_{0}=20$.

3.1.1. Single RHH cell. Consider the single cell with RHH dynamics,

$$
\begin{aligned}
& \frac{d V}{d t}=F(V, n, p), \\
& \frac{d n}{d t}=g(V, n) .
\end{aligned}
$$

Since the RHH model is a two variable model, it is useful to examine the dynamics in the phase plane. In Figure 2 the $V$-nullcline, a cubic-like shape, and the $n$-nullcline are plotted for a normal level of extracellular potassium, $p_{0}=20$. There is one steady state. At this parameter value the system is excitable, meaning that when the state of a cell is shifted quickly and sufficiently from its resting position, the state variables travel away from the steady state before returning to rest. The direction of flow across the nullclines is designated by the arrows in Figure 2.

The $V$-nullcline depends on $p$ through the potassium Nernst potential in this model. The effect of increasing $p$ on the $V$-nullcline is shown in Figure 3 (a). As $p$ increases, the lower knee of the $V$-nullcline raises, and with it, the steady state values of both $V$ and $n$ increase. The steady state transmembrane potential as a function of $p, V^{*}(p)$, is shown in Figure 3 (b). This steady state solution remains stable as $p$ increases until it undergoes a change in stability, a bifurcation. At this bifurcation point $P_{H_{1}}$, near $p \approx 27.5$, a pair of eigenvalues has zero real part and nonzero imaginary part. This bifurcation is a subcritical Hopf bifurcation. Beyond this point, the only stable solution to the system (3.1) is a large amplitude stable periodic orbit. As $p$ continues to increase, following the smallest steady solution $V^{*}(p)$, we reach a knee of the steady state curve, which is the first of two limit point bifurcations $(p \approx 31$ and $p \approx 24$ ). Eventually, for a sufficiently high $p$-value, the steady state regains its stability through a second, supercritical Hopf bifurcation at $p \approx 63.5$. The lower stable branch of the 


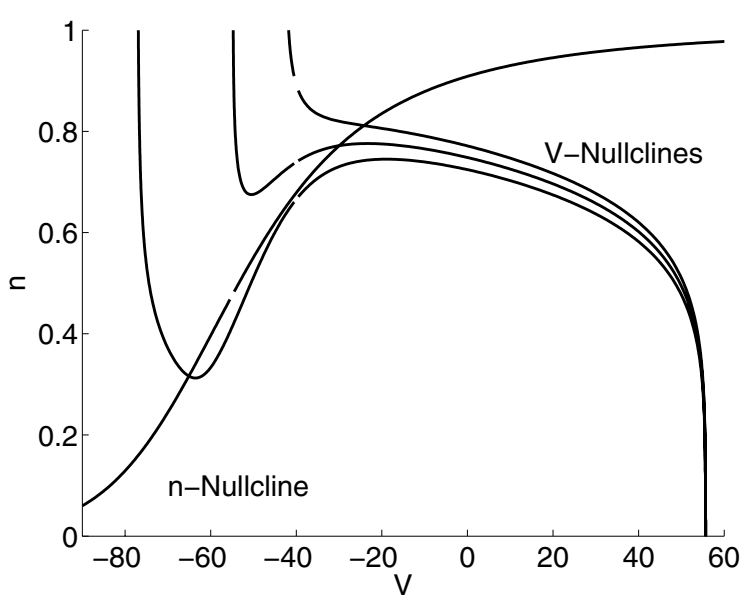

(a)

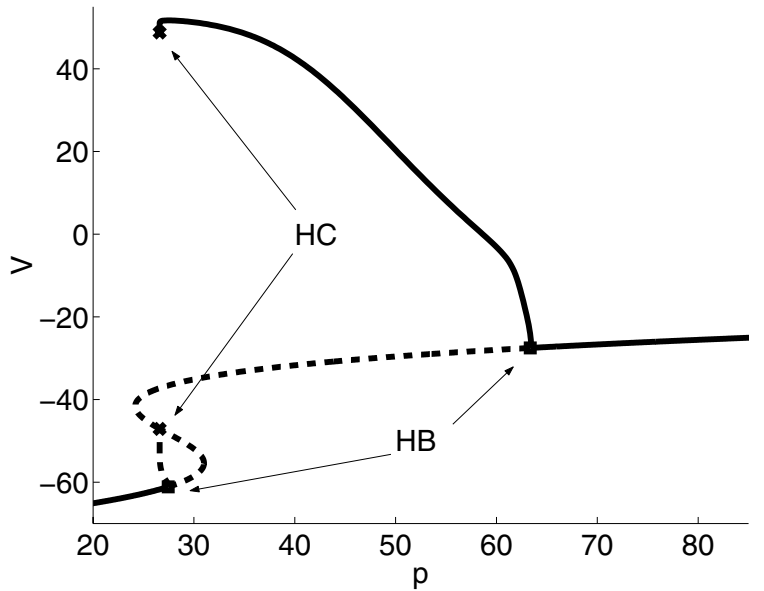

(b)

Figure 3. Effects of increasing $p$ on a single RHH model cell. (a) Shift in V-nullcline. This picture shows three different $V$-nullclines corresponding to different levels of extracellular potassium ( $p=20$ (normal), 45, and 80) for the RHH model. These show the depolarization of the transmembrane potential steady state as it depends upon p. (b) Bifurcation diagram. Increase of steady state transmembrane potential as it depends upon $p$ in an S-shape manner for the RHH model. The squares indicate Hopf bifurcations (HBs) ( $p \approx 27.5$ and $p \approx 63.5)$, where the steady state changes stability. The two curves emanating from the lower HB and upper $H B$ and ending at the homoclinic points (HCs) marked by $X$ 's are the amplitudes of the unstable and stable period orbits, respectively. There is a small region of overlap between the stable periodic orbit and the stable steady state on the lower branch yielding bistability.

S-shaped steady state curve represents a stable, excitable state, and the upper stable branch beyond the second Hopf point represents a stable, inexcitable state. The periodic solution that exists for intermediate $p$-values is a self-oscillatory solution. The region between the $p$-value where the stable periodic solution ends in a homoclinic orbit $P_{H C}(p \approx 26.5)$ and $P_{H_{1}}$ is a region of bistability where the stable periodic and stable steady state coexist.

Several qualitative features of ischemic tissue are reproduced by an increase in $p$. The resting transmembrane potential is elevated, and the action potential amplitude is reduced as shown in Figure 3 (b) by the decreasing amplitude of the self-oscillatory solution. For large enough $p$, the dynamics of the ischemic cell are completely inexcitable (even while the cell is not yet "dead" with $V=0$ ). The range of rest potential levels is appropriate for an ischemic cell even though the extracellular potassium levels are beyond physiological levels for cardiac tissue.

3.1.2. Coupled RHH cells. Now that we understand how the RHH dynamics of the single cell change with variation in $p$, we would like to understand how the coupling strength and mass of the individual cells affect the coupled cell dynamics. We couple two cells, one normal and one ischemic, each with RHH model dynamics. It is clear that if $p$ is elevated only slightly in the ischemic cell, regardless of the coupling strength or mass differential, both cells remain at resting states only slightly different from what would be their uncoupled resting states. 

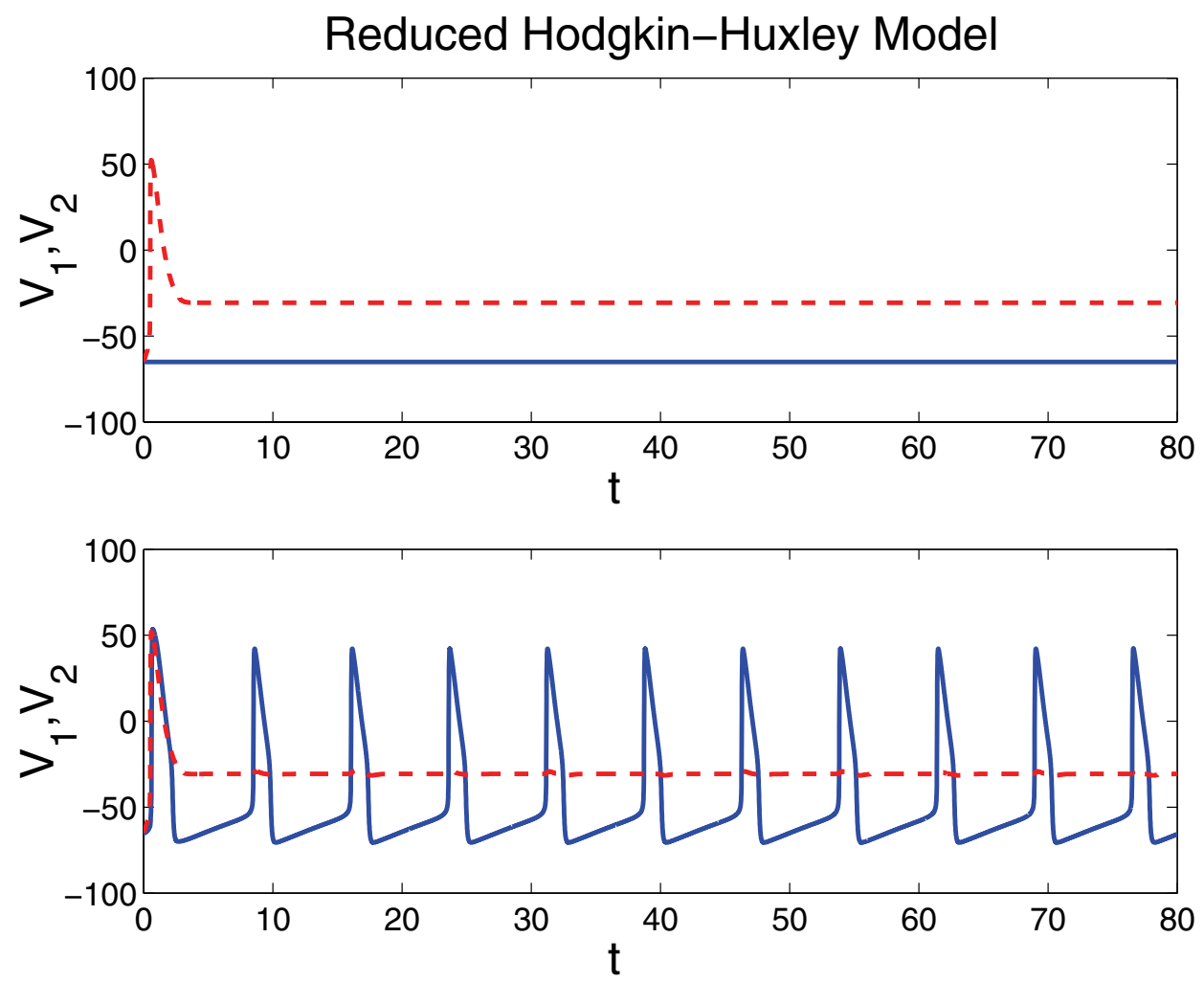

Figure 4. RHH model. The upper graph shows the steady states for the system if uncoupled (i.e., $\chi \delta=0)$ with $p=80$ in the ischemic cell. The lower graph shows the system coupled with $\chi \delta=0.15, p=80$, and $m=0.1$. The ischemic cell acts as a source of current to force the normal cell into oscillation. This feeds back to the ischemic cell causing imperceptibly small oscillations. The dashed lines are the ischemic cell's transmembrane potentials, while the solid line is the transmembrane potential for the normal cell.

This steady state is stable for the four state variable system.

Beyond this, however, the dynamics of the coupled system (2.3)-(2.6) are not immediately obvious. Evidence for interesting nontrivial behavior is shown in a time course plot for the coupled system with RHH ionics in Figure 4. Here, an ischemic cell of relative mass $1-m=0.9$ with $p=80$ is uncoupled (Figure 4 (a)) and then coupled (Figure $4(\mathrm{~b})$ ) by $\chi \delta=0.15$ to a normal cell of relative mass $m=0.1$ with $p_{0}=20$. When the two cells are uncoupled, both approach their respective stable steady states, but when coupled, the normal cell is forced by the current induced from the high resting potential of the ischemic cell, which oscillates ever so slightly around its uncoupled state.

The behavior in Figure 4 is different in the top and the bottom subfigures, and the only change has been to the composite coupling parameter, $\frac{\chi \delta}{m}$, causing a Hopf bifurcation. We define a curve in parameter space along which two of the eigenvalues of the coupled system (2.3)-(2.6) have zero real part and nonzero imaginary part as a Hopf curve. Figure 5 shows Hopf curves in $\frac{\chi \delta}{m}$ versus $p$ parameter space for two size parameters, $m=0.1,0.5$.

The shaded region is the set of parameter values at which oscillations are guaranteed. The solid curves, $\mathrm{HB}_{1}$ and $\mathrm{HB}_{2}$, are Hopf curves. The dotted curves, $\mathrm{LP}_{1}$ and $\mathrm{LP}_{2}$, are limit point 


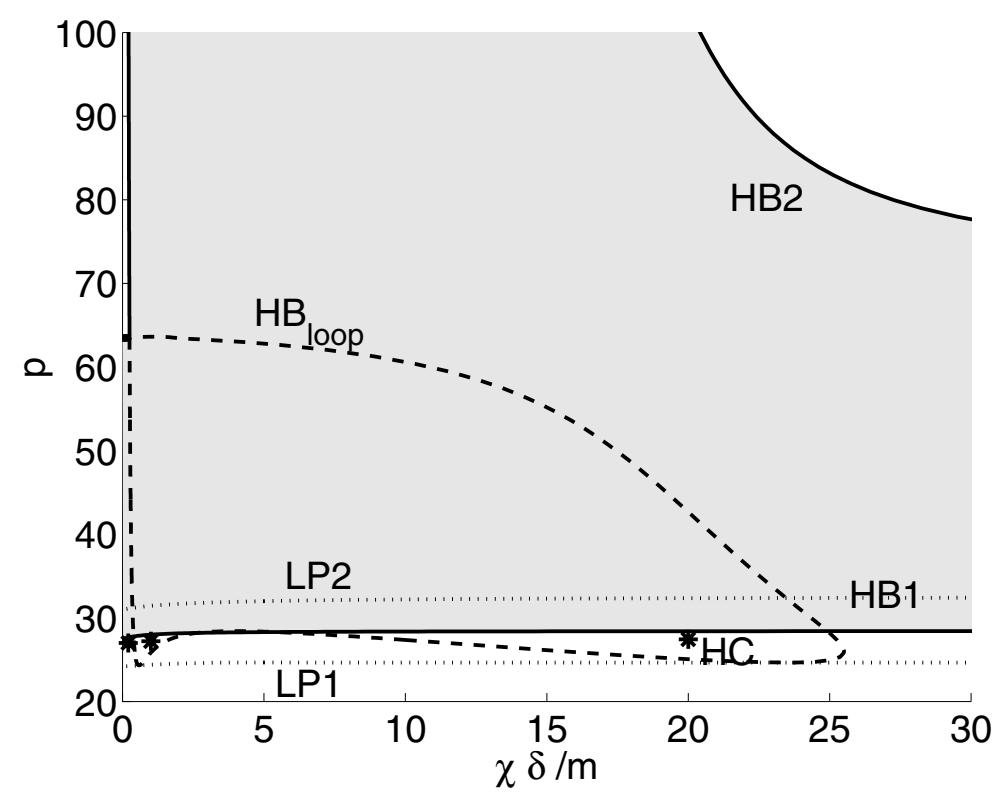

(a)

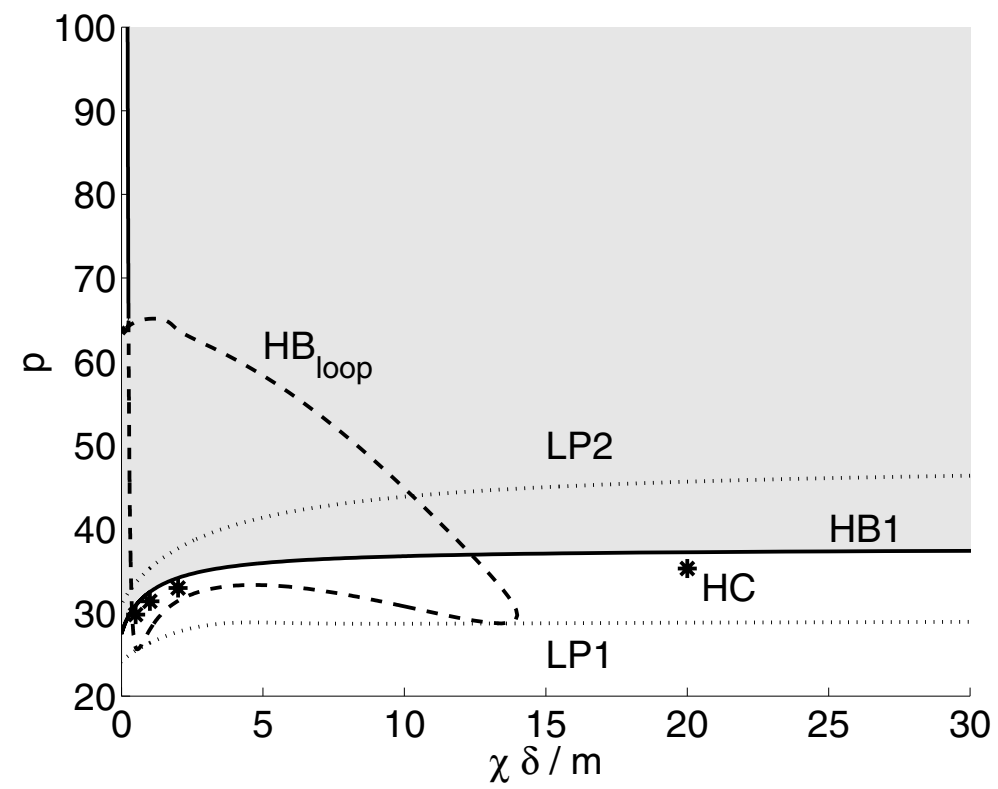

(b)

Figure 5. Unfolding diagrams for $\frac{\chi \delta}{m}$ versus $p$ at fixed $m=0.1$ and $m=0.5$. The shaded region is the set of parameter values at which oscillations are guaranteed. The solid curves, $H B_{1}$ and $H B_{2}$, are Hopf curves. The dotted curves, $L P_{1}$ and $L P_{2}$, are limit point curves. The dashed curve is a secondary Hopf curve. There is a curve of homoclinc bifurcations (which intersects the starred points). The points along this curve, one of which is labeled $H C$, are the p-values for a given $\frac{\chi \delta}{m}$ at which the periodic solution emanating from $H_{2}$ becomes homoclinic. Since $H C$ is below $H B_{1}$, between $H C$ and $H B_{1}$ in $p$ is a region of bistability. The lower Hopf curve, $H B_{1}$, and homoclinic points, $H C$, also shift upward for increasing $m$ except for being pinned at the p-axis. 
curves along which a real eigenvalue is zero. The dashed curve is a secondary Hopf curve. The Hopf curves and limit point curves were calculated using Auto97 bifurcation software [7]. The star points, one of which is labeled $\mathrm{HC}$, are the $p$-values for a given $\frac{\chi \delta}{m}$ at which the periodic solution emanating from $\mathrm{HB}_{2}$ becomes homoclinic. Since $\mathrm{HC}$ is below $\mathrm{HB}_{1}$ in $p$ for a given $\frac{\chi \delta}{m}$, between $\mathrm{HC}$ and $\mathrm{HB}_{1}$ is a region of bistability. Depending upon the initial conditions, the system approaches either the steady state solution or the periodic orbit. As $m$ increases, the upper Hopf curve, $\mathrm{HB}_{2}$, shifts outward (outside of picture frame) increasing the upper bound on the oscillatory region. The lower Hopf curve, $\mathrm{HB}_{1}$, and homoclinic points, $\mathrm{HC}$, also shift upward with increasing $m$ except for being pinned at the $p$-axis. This increases the lower bound on oscillations for each $\frac{\chi \delta}{m}>0$.

Looking more closely in a neighborhood of the $p$-axis in Figure 6, we see an interval in $p$ in which the uncoupled ischemic cell is self-oscillatory, $p \in\left[P_{o_{1}}, P_{o_{2}}\right]=[27.5,63.5]$. For small $m$ we expect the normal cell to have little effect on the ischemic cell so that as the coupling $(\chi \delta)$ increases we expect $\left[P_{o_{1}}, P_{o_{2}}\right]$ to remain an oscillatory region, which it indeed does (see Figure 6 (a)). Interestingly, for $p>P_{o_{2}}$ and coupling sufficiently large, we find another instability region which extends to the $\mathrm{HB}_{2}$ curve (see Figure 5 (a)). This region corresponds to oscillatory dynamics from two coupled cells that if uncoupled would each approach their stable rest states. Figure 4 shows an example of cells in such a parameter region.

As $m$ increases (see Figure $6(\mathrm{~b})$ ), the $\mathrm{HB}_{1}$ curve rises away from $\frac{\chi \delta}{m}=0$, indicating that the reduced ischemic cell needs higher $p$-values to begin oscillating. For $m$ large enough (see Figure $6(\mathrm{c})$ ) the $\mathrm{HB}_{1}$ curve coalesces with the $\mathrm{LP}_{2}$ curve and disappears above $\frac{\chi \delta}{m}$-values denoted by the Bogdonov-Takens bifurcation point, $\mathrm{BT}_{1}$. The boundary for the instability region is then the $\mathrm{LP}_{2}$ curve for $\frac{\chi \delta}{m}$ above $\mathrm{BT}_{1}$. (See Appendix $\mathrm{B}$ in [16] for further details.)

Figure 7 shows the time course for a relatively small ischemic cell $(m=0.1)$ coupled not all that strongly $(\chi \delta=0.455)$ to a normal cell that produces oscillatory dynamics in the normal cell. There are regions in coupling versus $p$ for a wide range of relative sizes $m$ which produce oscillations. Futhermore, a stable excitable normal RHH cell and a stable inexcitable RHH cell when coupled sufficiently strongly may produce large scale oscillations in the normal cell. The implication for large $m$ is that even a small amount of ischemia in a region sufficiently coupled with unaffected tissue may be sufficient to elicit extrasystolic action potentials independent of normal pacing. For small $m$ the ischemic cell remains at its resting potential while supplying a sufficient current to the normal cell to cause oscillations (see Figure 7).

3.2. Luo-Rudy I model. The Luo-Rudy I (LRI) model [15] is designed specifically for cardiac cell dynamics and includes many specific physiological features thereof. The state variables in the LRI model are the transmembrane potential, gating variables, and intracellular calcium. The dynamics are governed by eight differential equations for these state variables where $V$ is the transmembrane potential; $m, h$, and $j$ are sodium channel activating, fast inactivating, and slow inactivating gating variables, respectively; $d$ and $f$ are calcium channel activating and inactivating gating variables, respectively; $x$ is a potassium channel inactivating gating variable; and $C a_{i}$ is intracellular concentration of calcium. The governing equations governing are given in Appendix B.

3.2.1. Single LRI cell. We examine the single cell with LRI dynamics, 


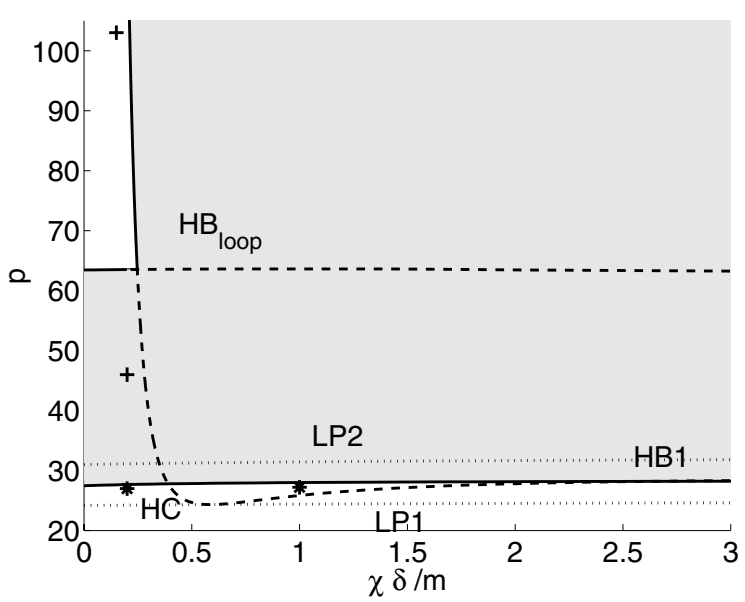

(a)

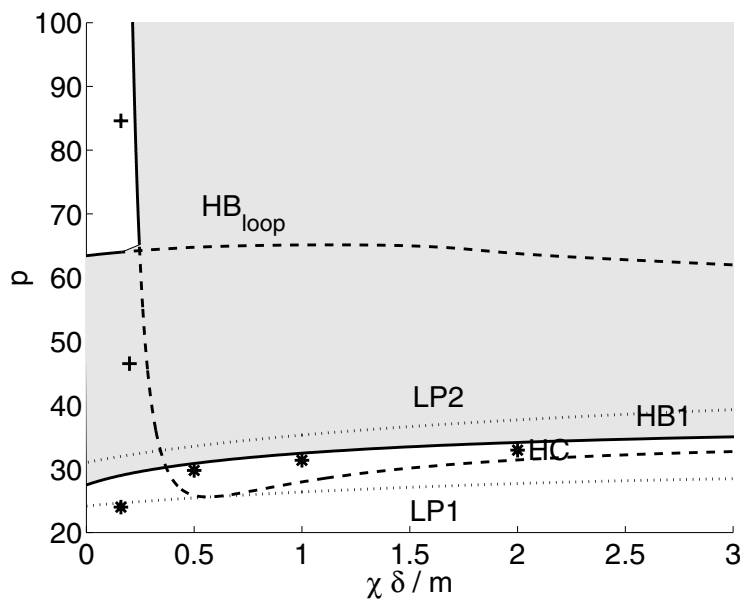

(b)

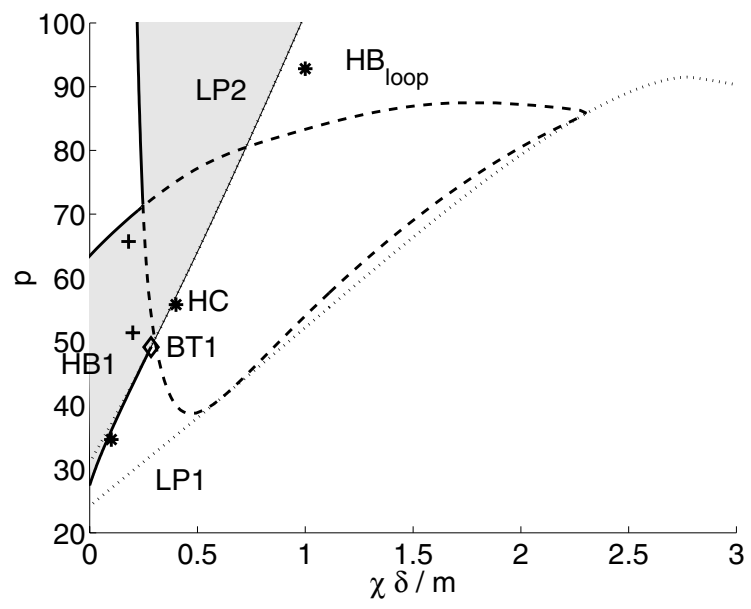

(c)

Figure 6. Unfolding diagrams for $\frac{\chi \delta}{m}$ versus $p$ at fixed $m=0.1, m=0.5, m=0.9$ zoomed into small $\frac{\chi \delta}{m}$. (a) and (b) focus on the region closer to the p-axis of Figure 5 (a) and (b), respectively. (c) For $m=0.9$, the slope of the $H B_{1}$ curve has dramatically increased compared to (a) and (b) and the curve ends in a BogdonovTakens, BT $T_{1}$, bifurcation. With the loss of the $H B_{1}$ curve, the boundary for purely oscillatory solutions becomes the limit point curve, $L P_{1}$. Between the $H C$ points and the $L P_{1}$ curve there exists a region of bistability. There is a curve of saddle-node of periodics bifurcations (which intersects the plus points). This creates a narrow strip for which there is bistability (either with a steady state or periodic solution). However, the basin of attraction is small for the emanating stable periodic orbit as not to affect the stability diagram appreciably. As coupling increases, the unstable periodic solution from the saddle-node of periodics interacts with the stable periodic that disappears in the homoclinic bifurcation. This initiates at the coupling value where the Hopf bifurcations cross transversally. 

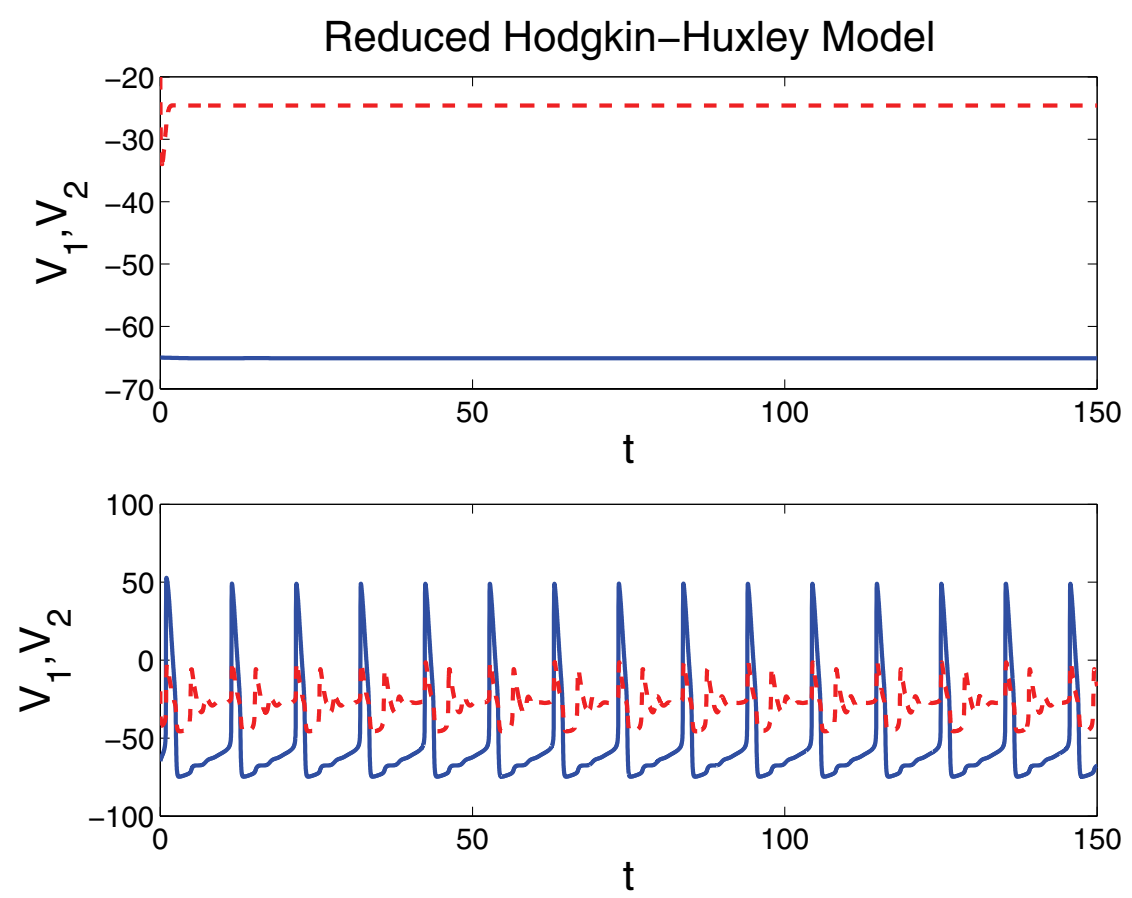

Figure 7. RHH model. The upper graph shows the steady states for the system if uncoupled (i.e., $\chi \delta=0)$ with $p=90$ in the ischemic cell. The lower graph shows the system coupled with $\chi \delta=0.455, p=90$, and $m=0.9$. The elevated resting transmembrane potential acts as a source of current to force the normal cell into oscillation which in turn perturbs the potential of the ischemic cell into a small amplitude oscillation about its elevated resting transmembrane potential. The dashed line is the ischemic cell transmembrane potential, while the solid line is the transmembrane potential for the normal cell.

$$
\begin{aligned}
& \frac{d V}{d t}=F(V, \vec{w}, p), \\
& \frac{d \vec{w}}{d t}=\vec{g}(V, \vec{w}),
\end{aligned}
$$

where $\vec{w}=\left[m, h, j, d, f, x, C a_{i}\right]^{T}$.

This particular physiological model has explicit dependence on $\mathrm{K}_{0}^{+}$through the potassium Nernst potential and the potassium channel conductance. The single cell steady state diagram for the LRI model is shown in Figure 8. The resting transmembrane potential depends monotonically on $p=\mathrm{K}_{0}^{+}$, and although it becomes more depolarized as $p$ increases, it never loses its stability (compare with the RHH steady state diagram Figure 3 (b)). Along with the increase in resting potential, the single ischemic cell also loses excitability as exhibited in Figure 9. The lack of self-oscillatory behavior of a single LRI cell for elevated $p$-values allows us to test whether or not this behavior is necessary for the coupled system to generate oscillations.

3.2.2. Coupled LRI cells. There is interesting behavior when two LRI model cells are coupled. Figure 10 (a) shows the uncoupled dynamics of the LRI model for $p=60$, while (b) shows the two cells from (a) coupled with $\chi \delta=0.005$ and $m=0.1$. In this example, there is 


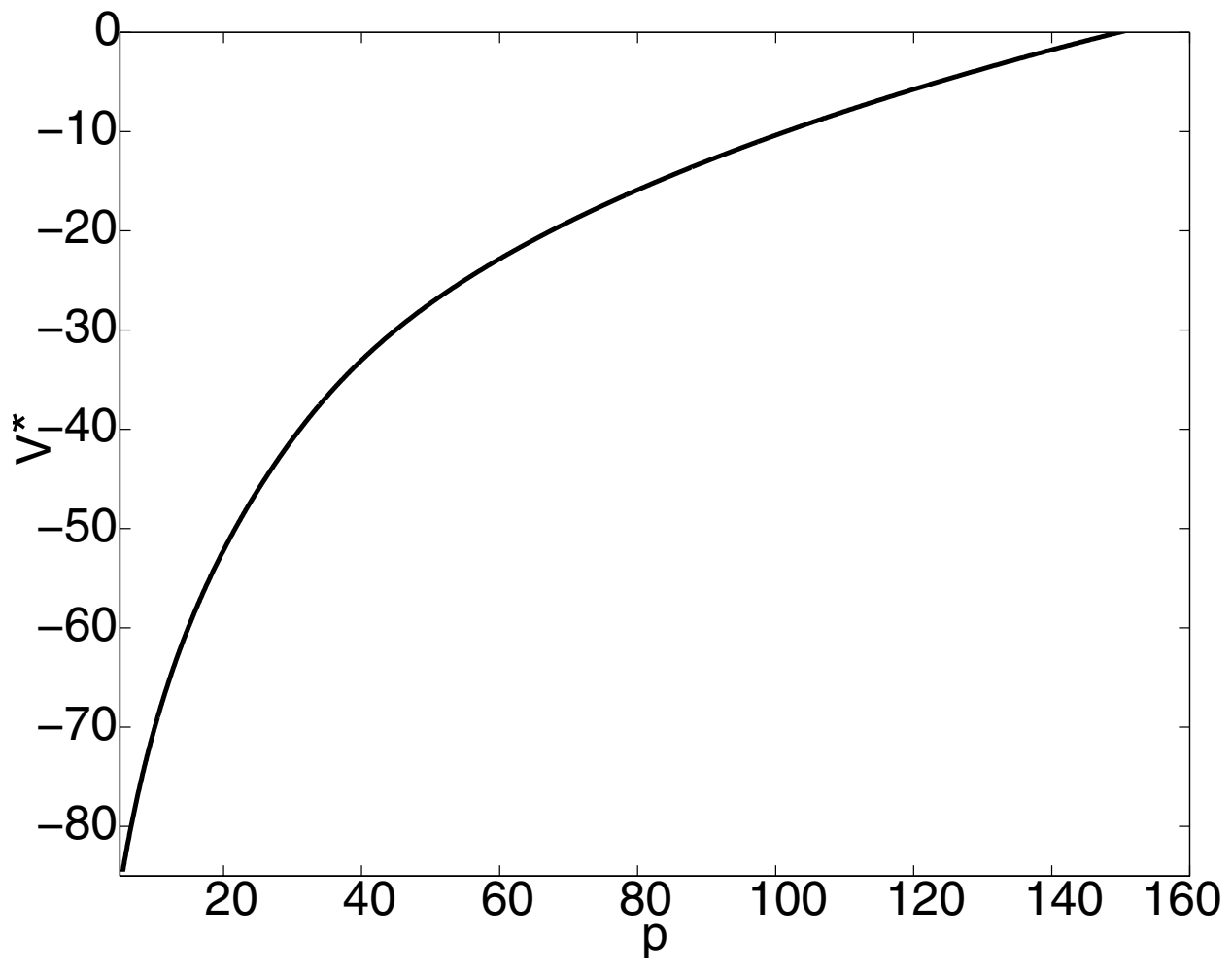

Figure 8. Steady state transmembrane potential as a function of $K_{0}^{+}$for the LRI model.

no self-oscillatory behavior in the ischemic cell so that the oscillation of the coupled system results from the coupling of the two cells.

To understand where in parameter space these oscillations occur we create unfolding diagrams. The unfolding diagrams in $p$ and coupling strength for the LRI model dynamics were found numerically using Auto97 bifurcation software [7]. These regions represent the parameter values at which ischemic cells couple to normal cells, both of which are otherwise stable, and induce oscillations. As shown in Figure 11, the enclosed oscillatory region slightly increases when $m$ is increased before collapsing as $m \rightarrow 1$ (not shown).

Figure 12 shows the transmembrane potentials of normal, $V_{1}$, and ischemic, $V_{2}$, cells along a one-dimensional slice through the unfolding diagram in Figure 11 (c). As $\frac{\chi \delta}{m}$ decreases for fixed $m=0.9$, the pair of Hopf points shift to higher $p$-values until they cross. Here the stable oscillation is lost and only the stable steady state remains for larger coupling. Uncoupled the normal cell has a steady state at $V_{1}=-84$ and the ischemic cell has the steady state curve as shown in Figure 8. However, for $\chi \delta=0.1$ and $m=0.9$ each of the resting potentials is affected. $V_{2}$ increases with increasing $p$ but at a lower rate compared to the uncoupled steady state, while $V_{1}$ increases as $p$ increases due to the coupling rather than remaining constant. For $p$ large enough a subcritical Hopf bifurcation gives rise to a rapid transition to stable oscillations in both cells. The amplitude of the stable periodic oscillation reaches a maximum in $V_{1}$ of about $-10 \mathrm{mV}$. These oscillations collapse as $p$ increases through a second, 


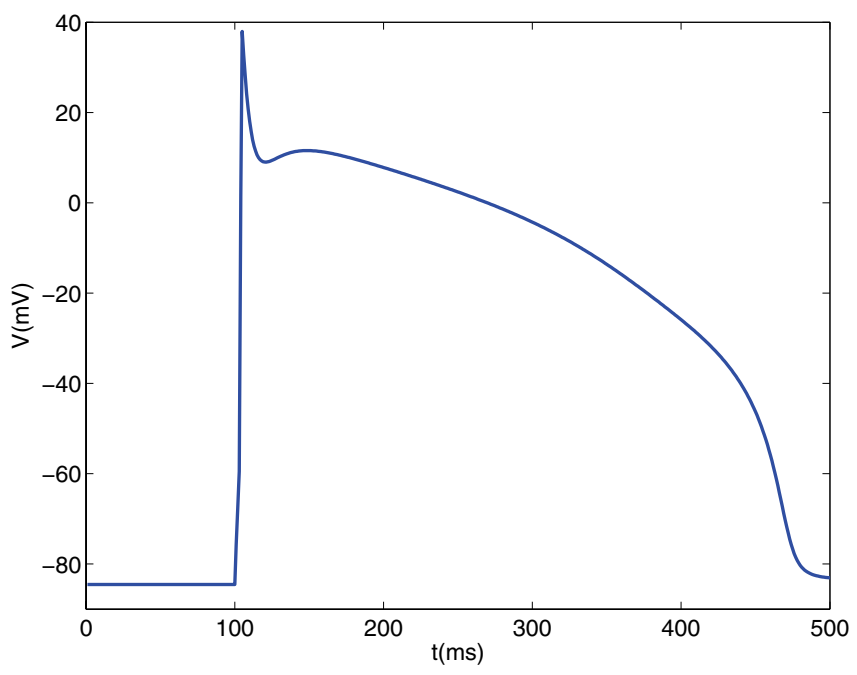

(a)

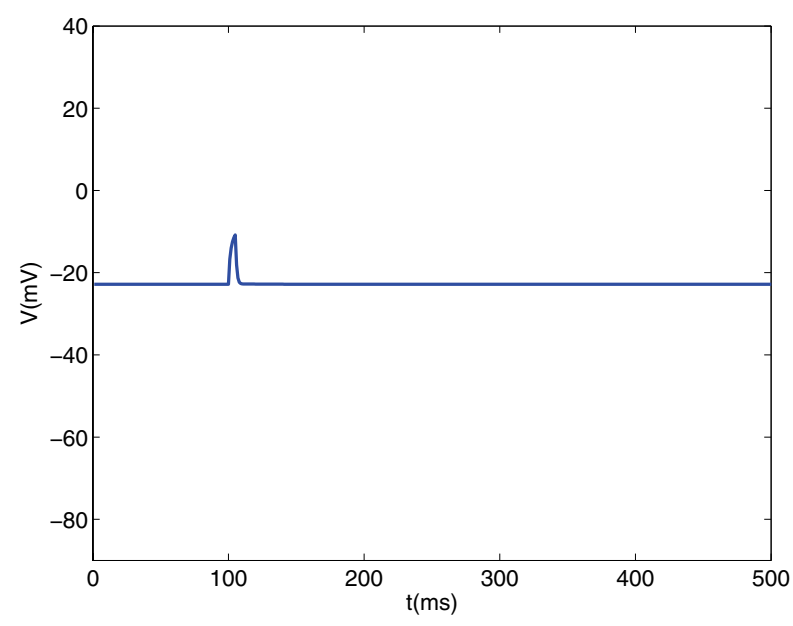

(b)

Figure 9. LRI model. (a) Result of a single $5 \mathrm{~ms}$ stimulus for normal cell parameters from resting conditions ( $\left.V^{*} \approx-84\right)$. (b) Result of a single $5 \mathrm{~ms}$ stimulus for elevated extracellular potassium $(p=60)$ from resting conditions $\left(V^{*} \approx-23\right)$. With elevated extracellular potassium, the cell is inexcitable and decays exponentially back to rest following the brief stimulus.

supercritical Hopf bifurcation. Since the unstable branch of periodic solutions emanating from the subcritical Hopf bifurcation exists in only a small neighborhood below the Hopf point, it is reasonable to take this Hopf point as a boundary (albeit approximate) in $p$ on significant oscillatory dynamics.

3.2.3. Comparison with experiments. To compare the oscillatory regions found from the LRI model quantitatively with experiments, we translate experimental conductance parameters into model parameters. Assuming an average cardiac cell has the dimensions $100 \mu \mathrm{m} \times$ 

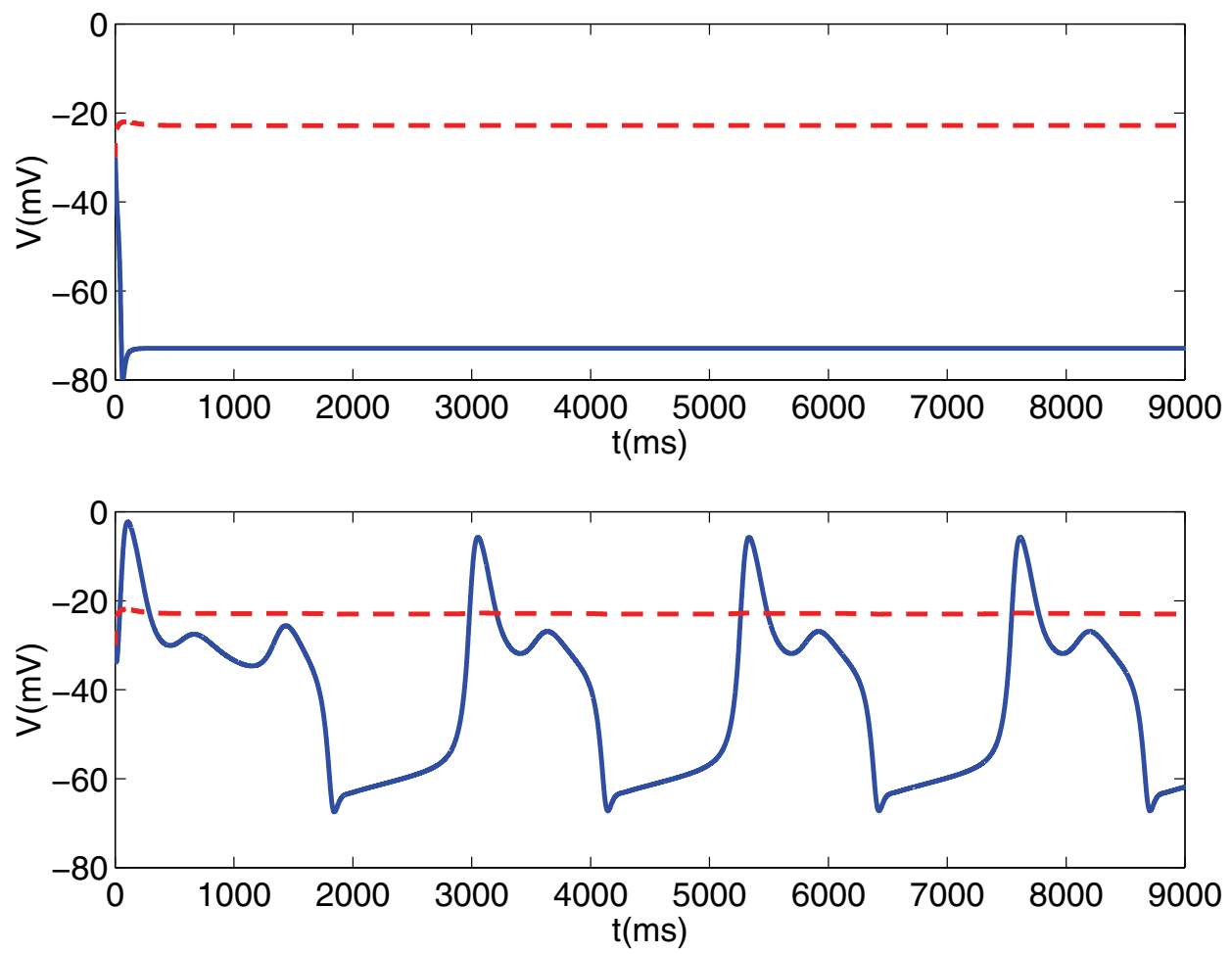

Figure 10. LRI model. The upper graph shows the steady states for the system if uncoupled (i.e., $\chi \delta=0$ ) with $K_{0}^{+}=60$ in the ischemic cell. The lower graph shows the system coupled with $\chi \delta=0.005, K_{0}^{+}=60 \mathrm{mM}$, and $m=0.1$. The ischemic cell effectively remains at its resting potential with only imperceptible oscillations, while it acts as a source of current to force oscillations on the normal cell.

$20 \mu \mathrm{m} \times 5 \mu \mathrm{m}$, then the average surface area for a cell is $5200 \mu \mathrm{m}^{2}$. Also assuming gap junctions are located only on the cell ends and cover a quarter of the surface area $\left(50 \mu \mathrm{m}^{2}\right)$, then $\chi$ (ratio of gap junctional surface area to total surface area) is about 0.01. Peters et al. [17] quantified the surface area covered by gap junctions per cell volume for normal human myocytes as $0.0051 \mu \mathrm{m}^{2} / \mu \mathrm{m}^{3}$. Using the typical cardiac cell dimensions yields the experimentally determined $\chi \approx 0.01$, which is consistent with our estimate.

Tan, Osaka, and Joyner [22] were unable to find oscillatory behavior when coupling a real VC to a passive model cell with an elevated (depolarized) RMP. They used a variety of coupling conductances $(0 \mathrm{nS}, 3 \mathrm{nS}, 5 \mathrm{nS})$ which correspond to $\chi \delta \approx(0,0.2,0.35)$ based on a resting membrane resistivity of $7 \times 10^{3} \Omega \mathrm{cm}^{2}$ and a total membrane area of $10^{4} \mu \mathrm{m}^{2}$ (roughly twice $\left.5200 \mu \mathrm{m}^{2}\right)$.

$$
\begin{aligned}
\chi \delta= & \frac{\text { gap junction area }}{\text { total surface area }} \times \text { gap junctional conductance } / \text { area } \\
& \times \text { resting membrane resistivity } \\
= & \frac{\text { gap junction conductance } \times \text { resting membrane resistivity }}{\text { total membrane area }} .
\end{aligned}
$$

The coupled cells were of similar size which corresponds to $m \approx 0.5$. So the coupling 


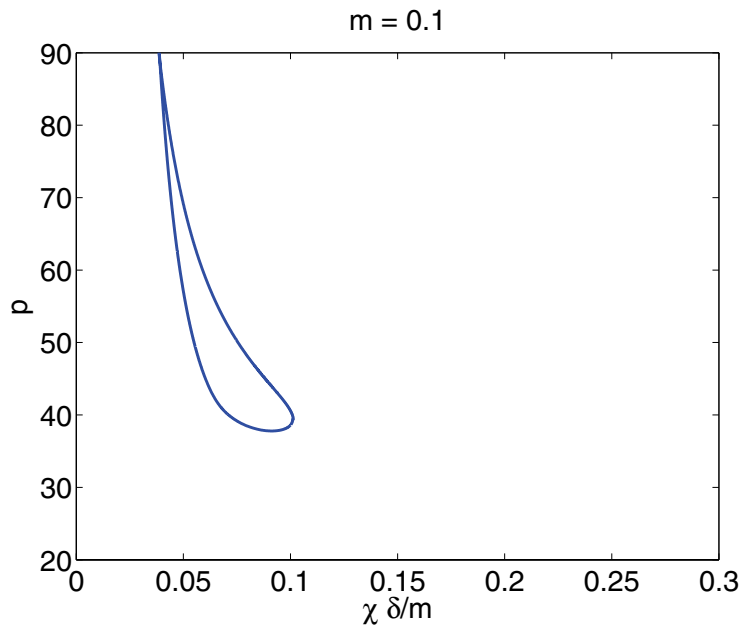

(a)

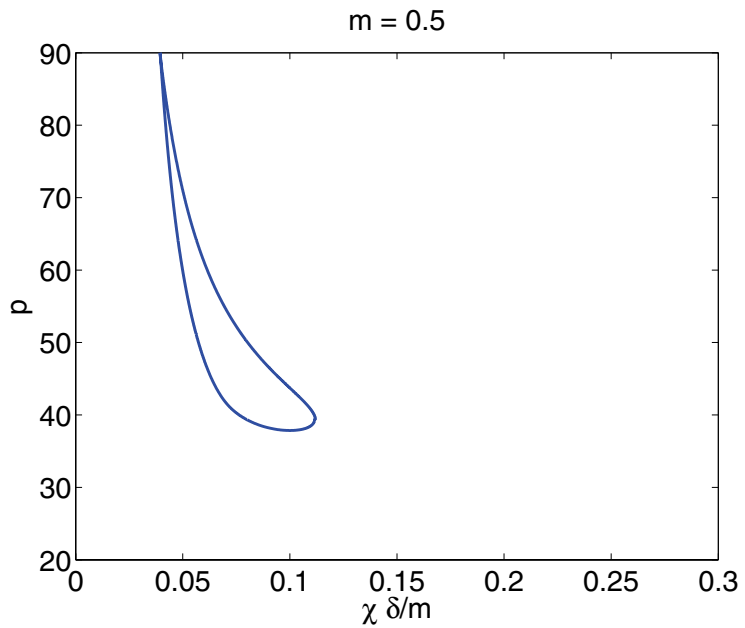

(b)

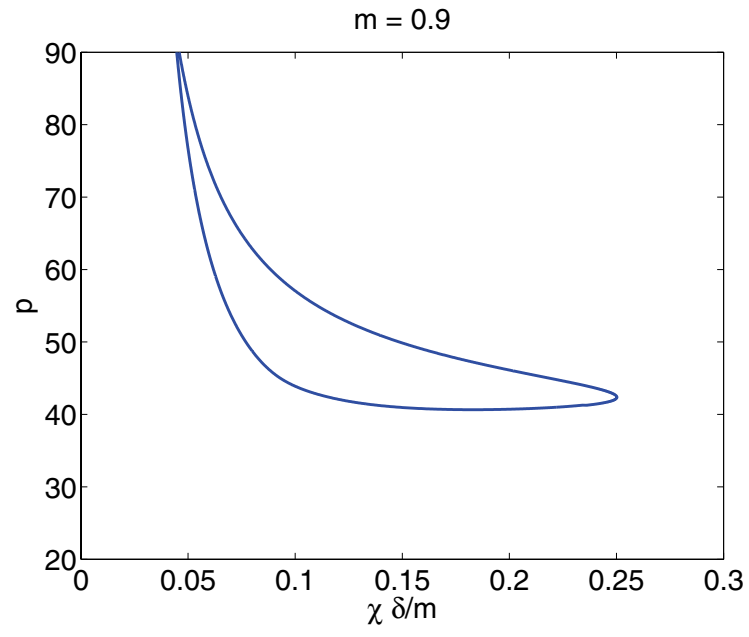

(c)

Figure 11. LRI model. Unfolding diagrams for $\frac{\chi \delta}{m}$ versus $p$ at fixed $m=0.1, m=0.5, m=0.9$.

conductances from [22] correspond to $\frac{\chi \delta}{m} \approx(0,0.4,0.7)$. The resting potentials for the passive model cell used were $(-20 \mathrm{mV},-10 \mathrm{mV}, 0 \mathrm{mV})$. Assuming the effect on $V_{2}^{*}$ from $V_{1}$ is negligible, $V_{2}^{*}$ corresponds to $p \approx 65$. In Figure $11(\mathrm{~b})$, the range of $\frac{\chi \delta}{m}$ with oscillatory behavior at $p=65$ is about $0.05<\frac{\chi \delta}{m}<0.06$ (coupling conductance between $0.36 \mathrm{nS}$ and $0.43 \mathrm{nS}$ ), and there is no oscillation for $p$-values corresponding to $V_{2}^{*}=0 \mathrm{mV}$ or $-10 \mathrm{mV}$. Since the oscillatory range for $V_{2}^{*}=-20 \mathrm{mV}$ is small and at small conductances, it is reasonable to conclude that this region was not found in experiments. 


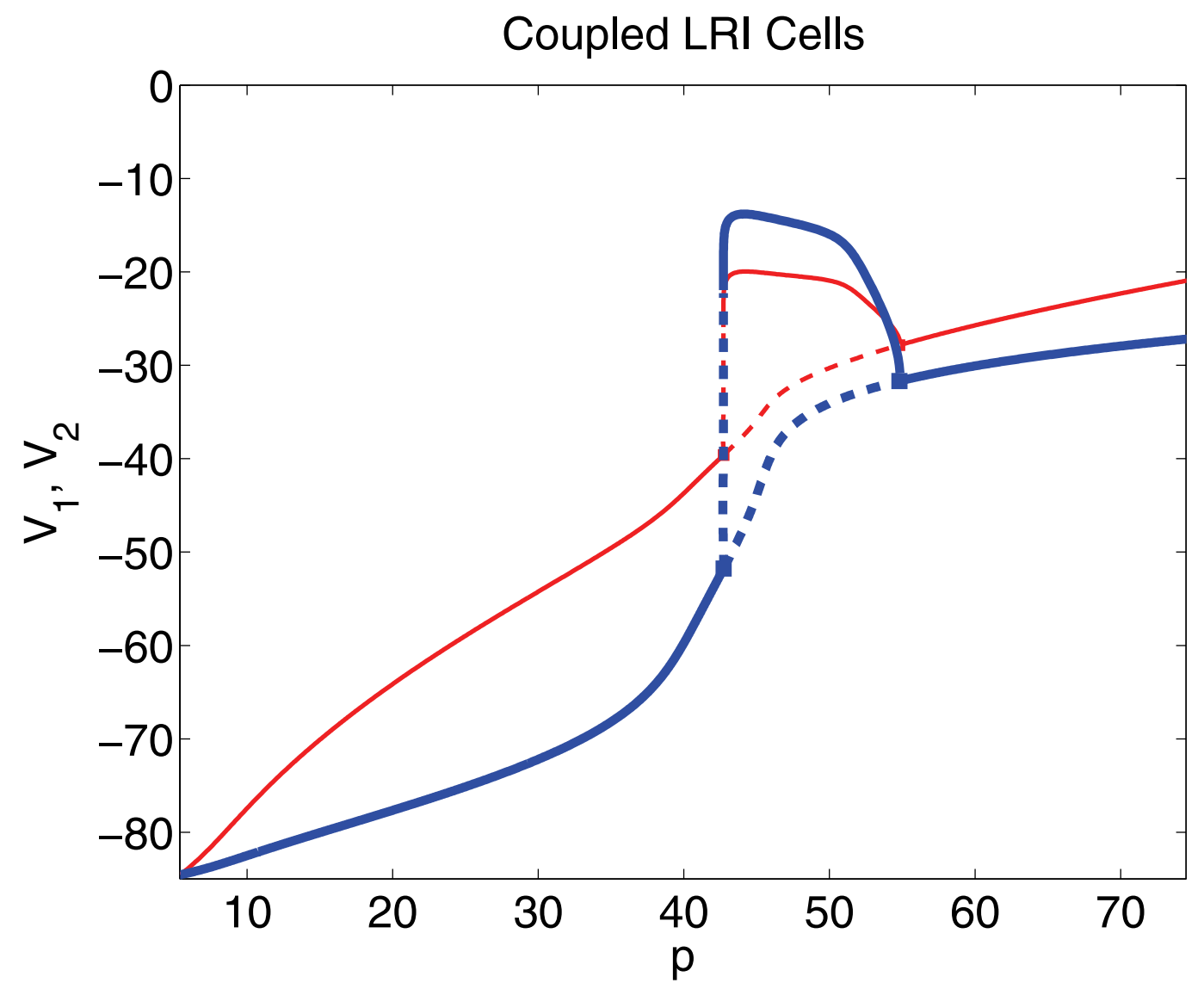

Figure 12. LRI model. Bifurcation diagrams for $p$ versus $V_{1}$ (thick, dark) and $V_{2}$ (thin, light) at $m=0.9$ and $\chi \delta=0.1$.

3.2.4. Calcium upstroke in coupled LRI system. The oscillations that are seen from the LRI model in Figure 10 are calcium-induced action potentials, that is, the calcium current produces the upstroke of the action potential rather than the sodium current. The sodium current and calcium currents are plotted in Figure 13. It seems reasonable then that enhancing the calcium handling with drugs as Kumar et al. [14] did would elicit calcium-induced action potentials. The effect of these drugs on the LRI model is not clear, but presumably they would act to expand the region of oscillation in $\frac{\chi \delta}{m}$ versus $p$ space, due to enhanced excitability of the calcium mechanism. Further discussion of this in relation to the mechanism of the calcium upstroke is found in section 5 .

4. Forced single cell to coupled cell dynamics. For small $m$, in both the RHH and LRI models the ischemic cell acts in one direction to send current to a normal cell without much return effect. The normal cell feels a constant depolarizing current and a constant conductance leak current. In this section we give a theory relating the forced oscillation of a single cell to oscillations of a coupled system. A technical theorem is stated in Appendix C. 


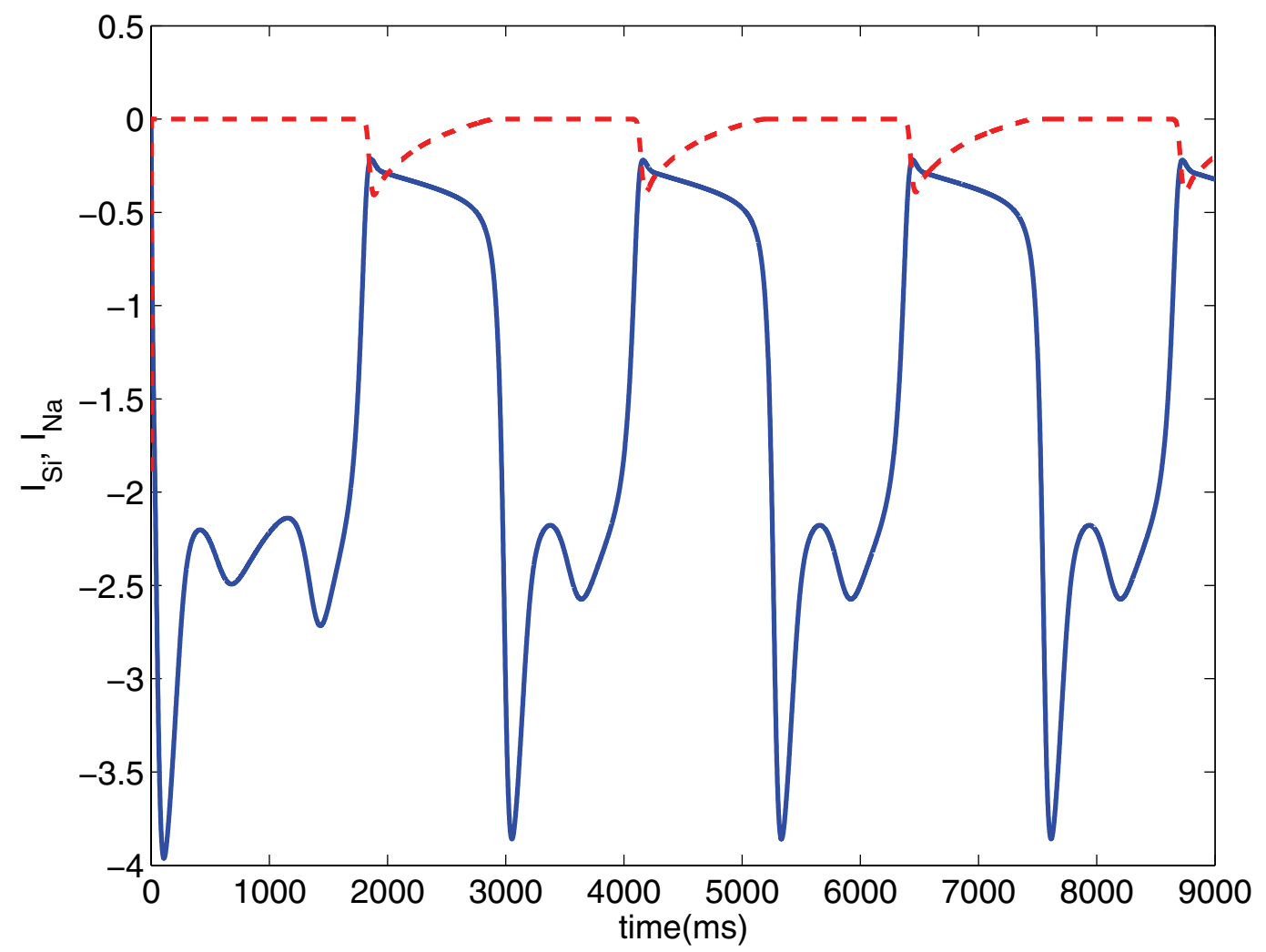

Figure 13. LRI model. The sodium (dashed) and calcium (solid) currents for the normal cell's potential time course in Figure 10.

Assumption A1. Suppose the system

$$
\begin{aligned}
\frac{d V_{1}}{d t}-F\left(V_{1}, \mathbf{w}_{1}, \mathbf{p}_{0}\right) & =-d V_{1}+I_{a p p}, \\
\frac{d \mathbf{w}_{1}}{d t} & =g\left(V_{1}, \mathbf{w}_{1}\right)
\end{aligned}
$$

exhibits oscillations in transmembrane potential for some $d$ and some $I_{a p p}$.

Assumption A2. Suppose the system

$$
\begin{aligned}
\frac{d V_{2}}{d t}-F\left(V_{2}, \mathbf{w}_{2}, \mathbf{p}\right) & =0, \\
\frac{d \mathbf{w}_{2}}{d t} & =g\left(V_{2}, \mathbf{w}_{2}\right)
\end{aligned}
$$

has a steady state $\left(V_{2}^{*}(\mathbf{p}), \mathbf{w}_{2}^{*}(\mathbf{p})\right)$ such that $d V_{2}^{*}(\mathbf{p})=I_{\text {app }}$.

Then the coupled system (2.3)-(2.6) for $\frac{\chi \delta}{m}=d$ exhibits oscillations in transmembrane potential provided $\frac{m}{1-m}$ is sufficiently small. If $V_{2}^{*}(p)$ increases as $p$ increases without bound and $d V_{2}^{*}\left(p_{0}\right) \leq I_{a p p}$, then there must exist a $p$ at which $d V_{2}^{*}(p)=I_{a p p}$. 


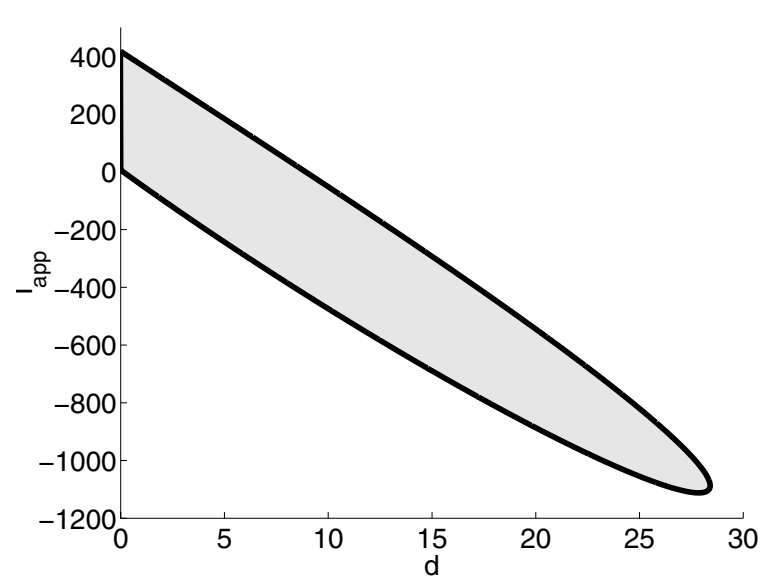

(a)

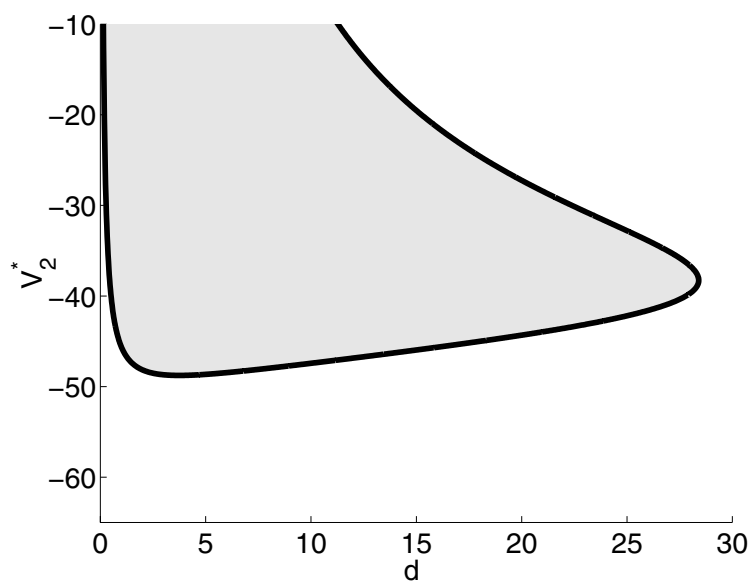

(b)

Figure 14. Unfolding diagram for the RHH single cell. (a) The Hopf bifurcation curve for the single RHH cell with changes in applied current, $I_{a p p}$, and leak conductance, d. (b) The Hopf bifurcation diagram for the single RHH cell with changes in fixed potential, $V_{2}^{*}$, and leak conductance, $d$. Subfigure (b) is obtained by plotting $d$ versus $V_{2}^{*}=I_{a p p} / d$. The shaded regions are parameter regions with oscillatory dynamics.

In other words, if applying a constant current and leak current with fixed leak conductance forces a normal cell into large scale oscillations, and if a second cell has a depolarized steady state transmembrane potential in the appropriate range, then the depolarized cell induces large scale oscillations in the normal cell. This occurs for an appropriate range of coupling conductances provided the normal cell is sufficiently small relative to the ischemic cell.

We now wish to verify that the theory applies to the two models described earlier. To verify the two assumptions A1 and A2, we consider the unfolding diagrams in the parameters $I_{a p p}$ and $d$ and the unfolding diagrams in the parameters $V_{2}^{*}=I_{a p p} / d$ and $d$ for the two sets of ionics. Figure 14 (a) shows that a single cell with RHH dynamics has oscillations for a region in $d$ versus $I_{a p p}$ satisfying Assumption A1. Figure 14 (b) shows where $d$ versus $V_{2}^{*}=I_{a p p} / d$ yields oscillations so that if the RHH steady state of equations (4.2) is located in the shaded region, Assumption A2 is satisfied. From Figure 3 we see there is a range of $p$-values with $V_{2}^{*}$ in the shaded region for which the theory applies in which oscillations exist in the coupled system (2.3)-(2.6).

Similarly, for LRI dynamics, Figure 15 (a) shows a region in $d$ versus $I_{a p p}$ where oscillations satisfying Assumption A1 occur. Figure 15 (b) shows where $d$ versus $V_{2}^{*}=I_{a p p} / d$ yields oscillations so that if the LRI steady state of equations (4.2) is located in the shaded region, Assumption A2 is satisfied. Figure 8 shows a range of $p$-values for which $V_{2}^{*}$ is in the shaded region of Figure $15(\mathrm{~b})$.

For sufficiently small $m$ the coupled system should behave similarly to the single cell under appropriate forcing. In Figure 16, we compare the single cell bifurcation diagram of Figure 14 (b) with the bifurcation diagram for the coupled cells from Figure 5 (a). There are two mechanisms by which the coupled cells may become unstable. The first has already been 


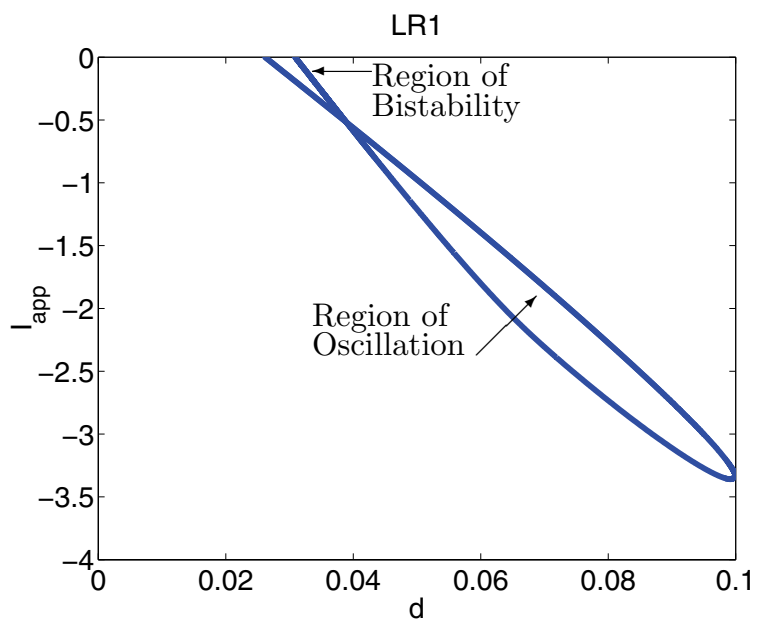

(a)

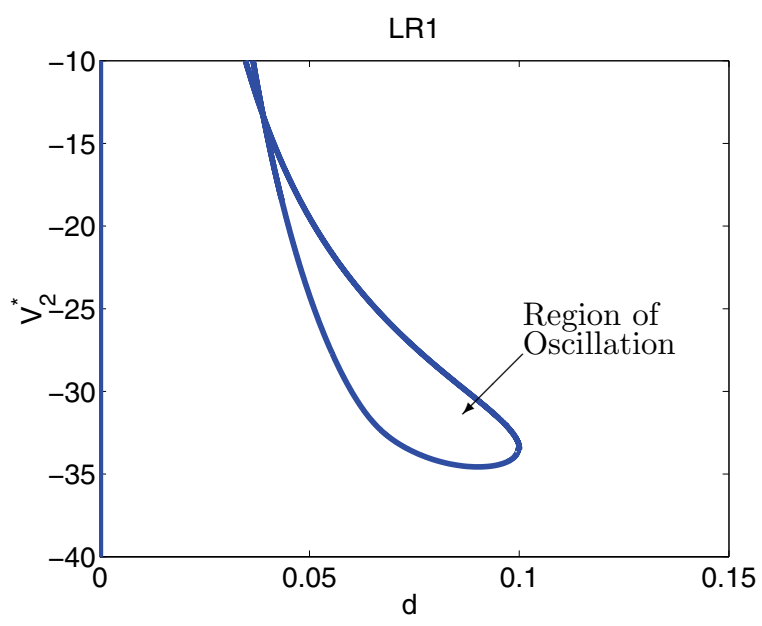

(b)

Figure 15. Unfolding diagram for the LRI single cell. (a) d versus $I_{a p p}$ for the LRI model. Inside the closed region the system is oscillatory. (b) d versus $V_{2}^{*}=I_{a p p} / d$.

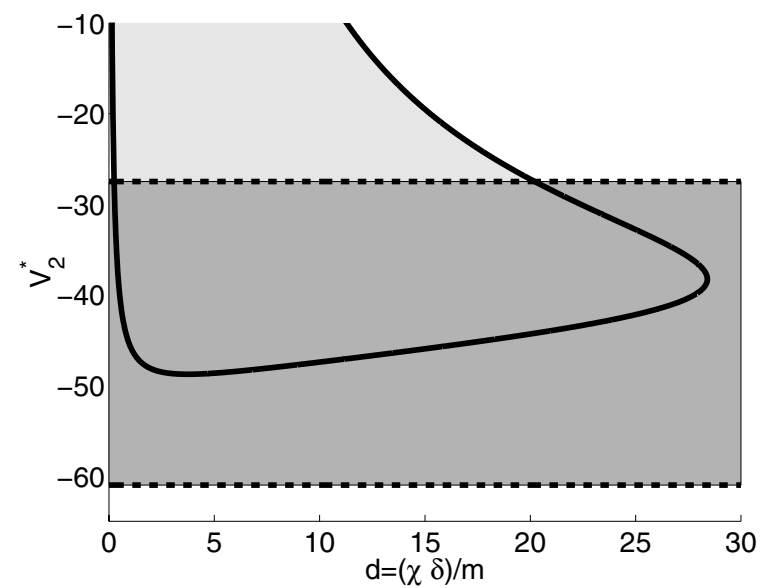

(a)

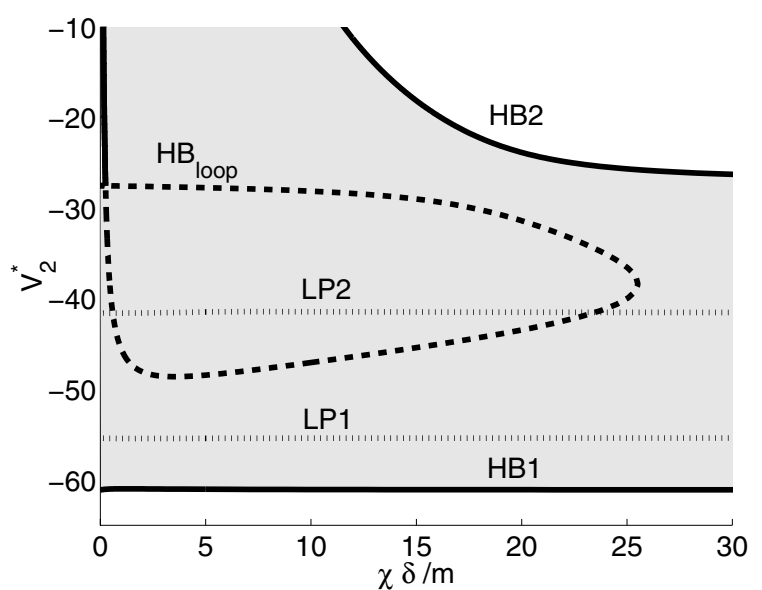

(b)

Figure 16. Comparison between the oscillatory region of a single cell and that of the coupled system for the RHH model. (a) The single cell bifurcation diagram as in Figure 14 (b) but with dashed lines added. The ischemic cell is self-oscillatory when its rest potential lies between the dashed lines. (See text.) (b) The coupled cell bifurcation diagram as in Figure 5 (a).

described as a forcing on a normal cell. The second mechanism is from the self-oscillation of the ischemic cell which may occur upon elevation of $p$. The dashed lines in Figure 16 (a), also denoted by the Hopf bifurcation squares in Figure 3 (b), are those transmembrane potentials 


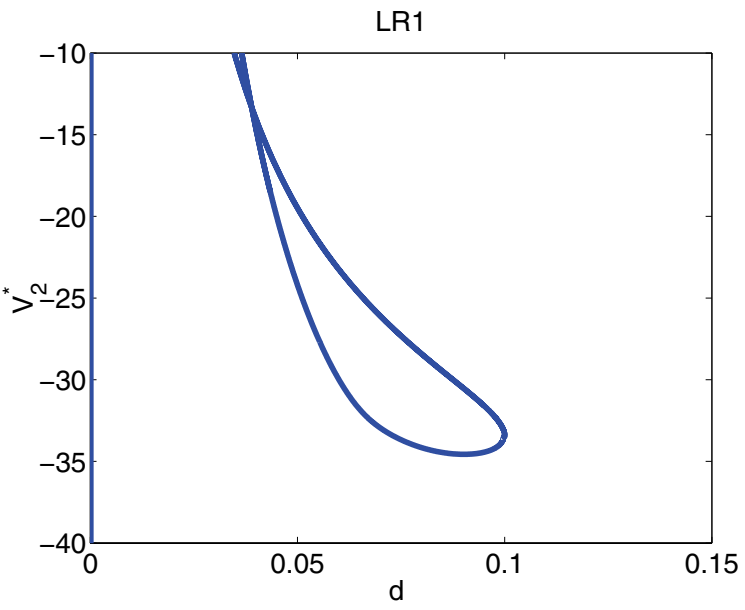

(a)

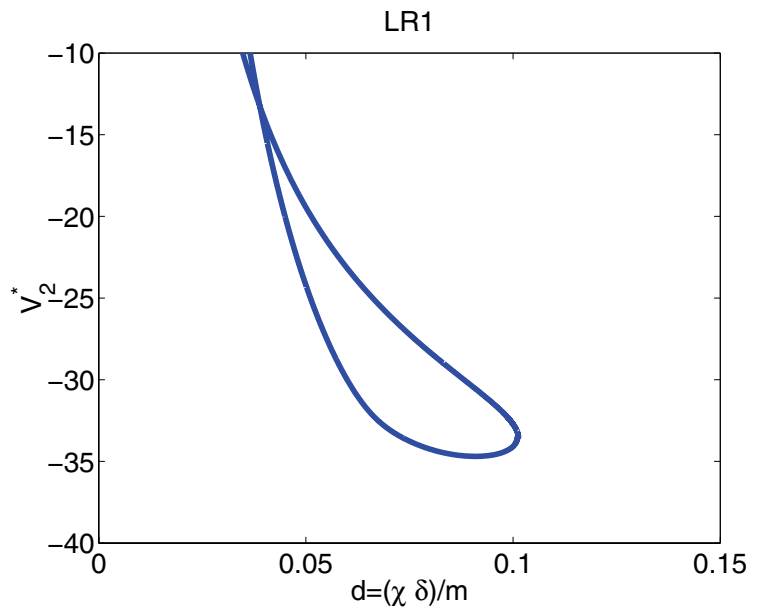

(b)

Figure 17. Comparison between the region of oscillation based on the applied and leak currents and the coupled system with $m=0.1$ in the LRI model. (a) The forced single cell oscillatory region. (b) The coupled system oscillatory region.

between which the ischemic cell oscillates when uncoupled from the normal cell. The oscillatory regions in Figure 16 (a) and (b) are remarkably similar, as expected.

As shown in Figure 17 for the LRI model, there is an almost exact correspondence between the curve derived from the single system in Figure 17 (a) and the curve obtained with the coupled systems and small $m(m=0.1)$ in Figure $17(\mathrm{~b})$. These results confirm the validity of the theory.

\section{Discussion.}

5.1. Calcium current. We have shown that excitable cells oscillate for certain ranges of applied and leak currents, but the morphology of the oscillations in the LRI model is distinct from those induced by a periodic current stimulus. This difference in morphology stems from the suppression of the inward sodium current during a fixed applied current so that the action potentials are driven only by the inward calcium current.

The fact that the oscillations in the coupled cell LRI model are driven by calcium can be understood from the steady state gating dependence on the transmembrane potential. In Figure 18 the infinity curves for $m, h, d$, and $f$ are plotted. At the normal steady state, the $m$ gate is closed, while the $h$ gate is open. Following a stimulus that suddenly raises the transmembrane potential, the $m$ gate opens rapidly with time constant $\tau_{m}$, while the $h$ gate begins to close slowly with a time constant $\tau_{h}$. Early in the action potential the $h$ gate closes shutting off the sodium current. As the transmembrane potential begins to rise from the sodium current, the potassium and calcium gates are opened on a slower time scale. The $d$ activation gate of the calcium channel begins to open slowly, while the $f$ inactivation gate closes even more slowly, allowing a calcium current. Gate responses of potassium channels 

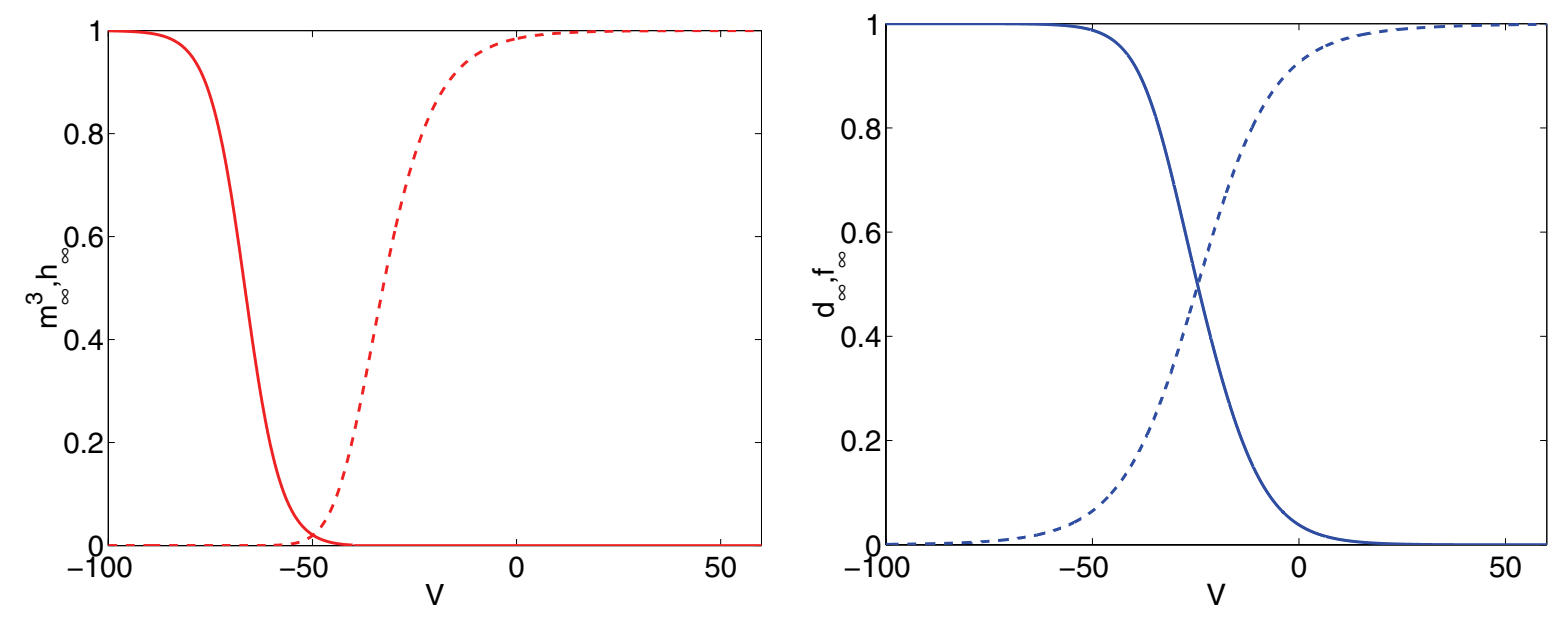

Figure 18. Infinity gating curves for the LRI model. (a) $h_{\infty}$ is the solid curve, while $m_{\infty}$ is the dashed curve. The $j_{\infty}$ curve lies almost exactly on the $h_{\infty}$ curve. (b) $f_{\infty}$ is the solid curve, while $d_{\infty}$ is the dashed curve.

are activated and potassium flows out of the cell in an outward current. The slow change in the balance between the inward calcium current and outward potassium current makes up the rest of the action potential in the LRI model. Finally, the transmembrane potential returns to rest where the gates reset.

Under the conditions studied here with a normal cell coupled to a cell with elevated resting transmembrane potential, the dynamics of the normal gates are altered. The resting transmembrane potential is raised slowly in the normal cell due to electrotonic coupling. If the resting transmembrane potential of the normal cell is raised above $-55 \mathrm{mV}$, then the sodium current is never triggered, because the $h$ gate is never opened. On the other hand, the calcium curves, $d_{\infty}$ and $f_{\infty}$, provide a window where both gates are open for an interval of $V$. For the oscillation shown in Figure 10, the rest potential of the normal cell is raised to about $-55 \mathrm{mV}$ closing the $h$ gate while the $m$ gate remains closed, but at the same time the $d$ gate is opened while the $f$ gate is also open, which activates the calcium current. This is the calcium current that causes the autocatalytic increase in $V$.

The $m_{\infty}$ and $h_{\infty}$ curves for the RHH model are as shown in Figure 19. Since $h_{\infty}$ is approximated by $0.85-n_{\infty}$, the $h_{\infty}$ curve does not asymptote to 1 or 0 as $V$ gets large negative or large positive, respectively. However, it is clear that there is a substantial window where both $m$ and $h$ are activated. This leads to a sufficient sodium current to achieve threshold and allow for an oscillatory action potential at elevated rest potentials.

The LRI model does not have a sodium based upstroke at high RMPs but does allow for calcium to play that role, while the RHH model has sodium upstrokes at higher resting potentials. It is not clear that these represent actual differences between cardiac cells and neural cells or are merely differing features of these models.

The effect of calcium handling drugs such as BAY K 8644 used in the experiments of Kumar and Joyner [13] is meant to be like a sympathetic nervous stimulation. The study 


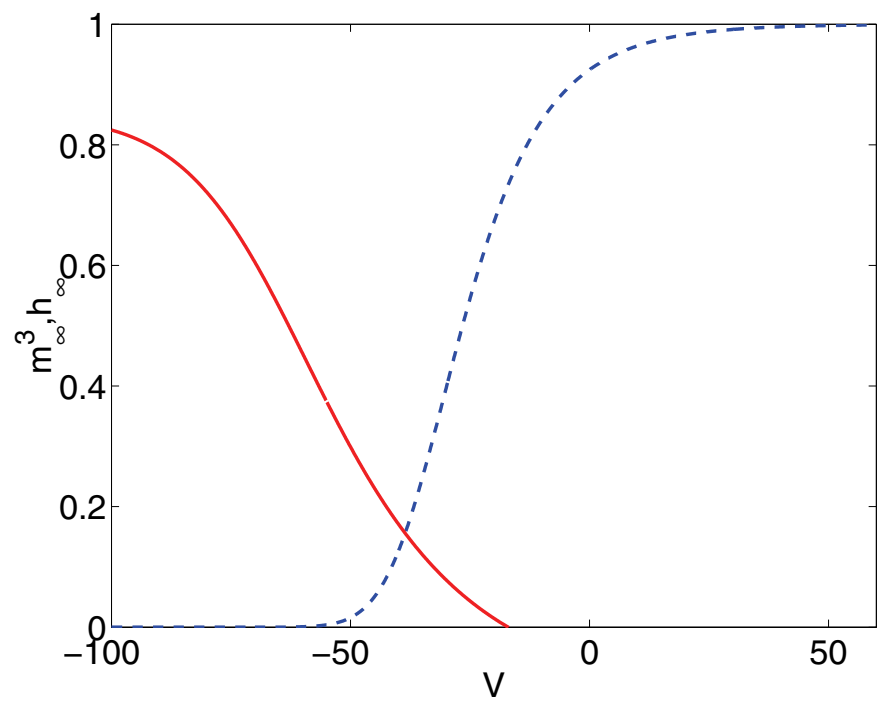

Figure 19. The infinity curves for the RHH model. $h_{\infty}$ is the solid curve, while $m_{\infty}$ is the dashed curve.

of Kumar and Joyner assessed the effect of these types of drugs on normal cells coupled to depolarized cells. They found that enhancing the calcium handling of normal cells and coupling them to depolarized cells induced EADs - EADs that Tan and Joyner could not find. As in Kumar and Joyner, we "add" this effector to the normal cell and couple it with an ischemic cell. The enhancement of the calcium handling by BAY K 8644 changes the position of the gating curves. According to Adachi-AkaHane, Cleeman, and Morad [1], "the $\mathrm{Ca}^{2+}$ channel agonist (-)-BAY K 8644 enhanced $\mathrm{Ca}^{2+}$-channel current $\left(\mathrm{I}_{\mathrm{Ca}}\right)$, shifted the activation curve by $-10 \mathrm{mV}$, and significantly delayed its inactivation." A shift of the activation curve by $-10 \mathrm{mV}$ is accomplished in the LRI model by shifting $d_{\infty}(V)$ to $d_{\infty}\left(V-V_{\text {shift }}\right)$, where $V_{\text {shift }}=-10 \mathrm{mV}$. The result is a larger window beneath the $d_{\infty}$ and $f_{\infty}$ curves, implying a larger window current (see Figure 20).

Figure 21 shows a closed region for the $d_{\infty}$-shifted system in which the only solution is oscillatory. Compare this region to Figure 11 (b). The oscillatory solution parameter region for the $d_{\infty}$-shifted system is found at much lower, more physiological $p$-values with the range of $p$-values greatly reduced. The coupling strength at which the system oscillates is also higher. The amplitude and baseline potentials of the oscillatory solution seen in Figure 22 correspond qualitatively with EADs in Kumar and Joyner. Unlike the unshifted system, as coupling decreases below the enclosed parameter region of oscillatory solutions, the region of bistability between stable steady state expands (see Figure 23). The potential for important hysteretic behavior is available. And, like the RHH model (cf. Figure 5 at HC points), in a narrow neighborhood (not shown) just below the lower Hopf curve (outside of the region of oscillation) bistability exists between a stable oscillatory solution and a stable steady state. Oscillatory solutions exist only for very low levels of $p$ for small coupling. However, it is not clear how large the basin of attraction is for this solution.

Using the theoretical adjustment implied by the experimental drug BAY K 8644, we have found a region in parameter space which may be related to the EADs observed in the 


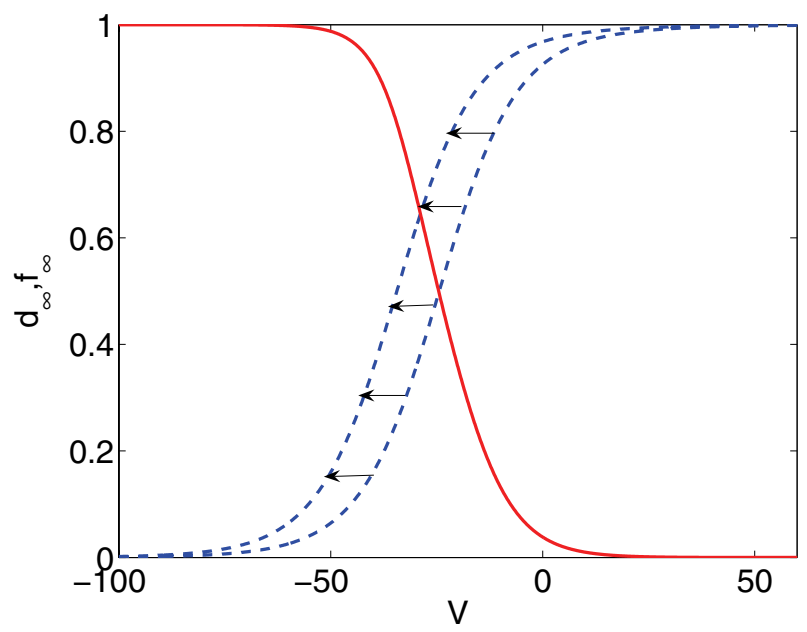

Figure 20. The infinity curves for the LRI model. The effect of BAYK 8644 is a negative shift of the activation curve, $d_{\infty}$ (dashed), by $-10 \mathrm{mV}$.

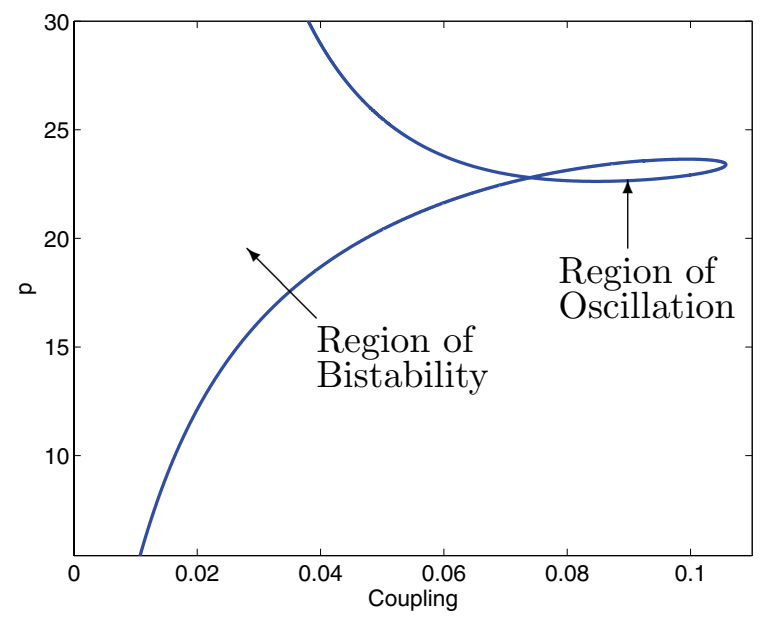

Figure 21. The LRI model with $d_{\infty}$-shift. The closed region is where there are only oscillatory solutions.

Kumar and Joyner work. This suggests that reducing the coupling and increasing the ischemia parameters in the drug-free experiment (see Figure 5 (b)) should produce the oscillations that Tan and Joyner were unable to find.

5.2. Reperfusion. Even if arrhythmias do not occur during the onset of ischemia, there is a likelihood of arrhythmias occurring upon reperfusion such as observed in the Picard and Rouet experiments $[18,20]$. We have shown that there exists a region in parameter space where the solution to (2.3)-(2.6) is oscillatory. However, during an ischemic event the parameters are dynamic. Figure 24 shows a potential trajectory through parameter space consistent with 


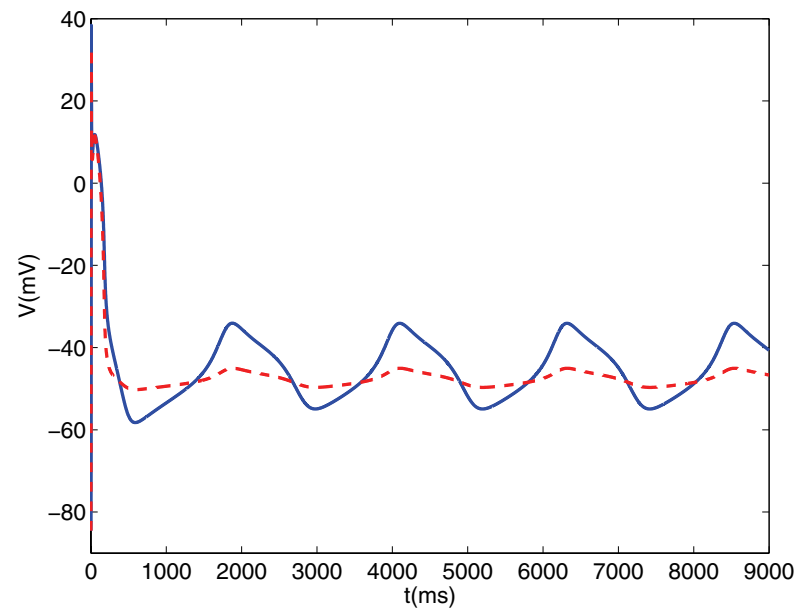

Figure 22. The LRI model with $d_{\infty}$-shift. The time course initiated from normal steady state shows oscillations in the nonischemic drug affected region (-) and the ischemic region (- -). Coupling is 0.09 and $p=23$ for equal cell mass.

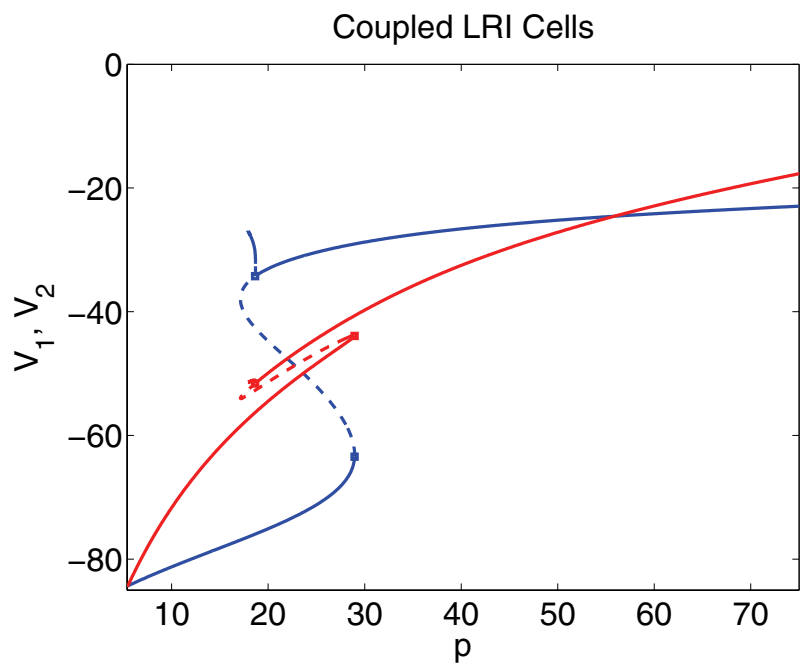

Figure 23. Bifurcation diagram for the coupled LRI model cells with $d_{\infty}$-shift in the normal cell for coupling of 0.04. $V_{1}$ is the $S$-shaped curve. Between the Hopf bifurcations there is a region of bistability.

changes in the parameters on the appropriate relative time scales.

Beginning at normal conditions there is no ischemia and coupling is relatively strong. After the onset of ischemia, the level of ischemia rises while the coupling remains constant. ${ }^{1}$ The degree of ischemia then plateaus. For example, recall the trajectory of extracellular potassium during ischemia: a rapid rise is followed by a plateau followed by a slow gradual

\footnotetext{
${ }^{1}$ Coupling is related to the degree of ischemia, but the difference in time scales of the uncoupling of gap junctions as compared to other chemical results of ischemia (i.e., extracellular potassium increase) permits the independence assumption.
} 


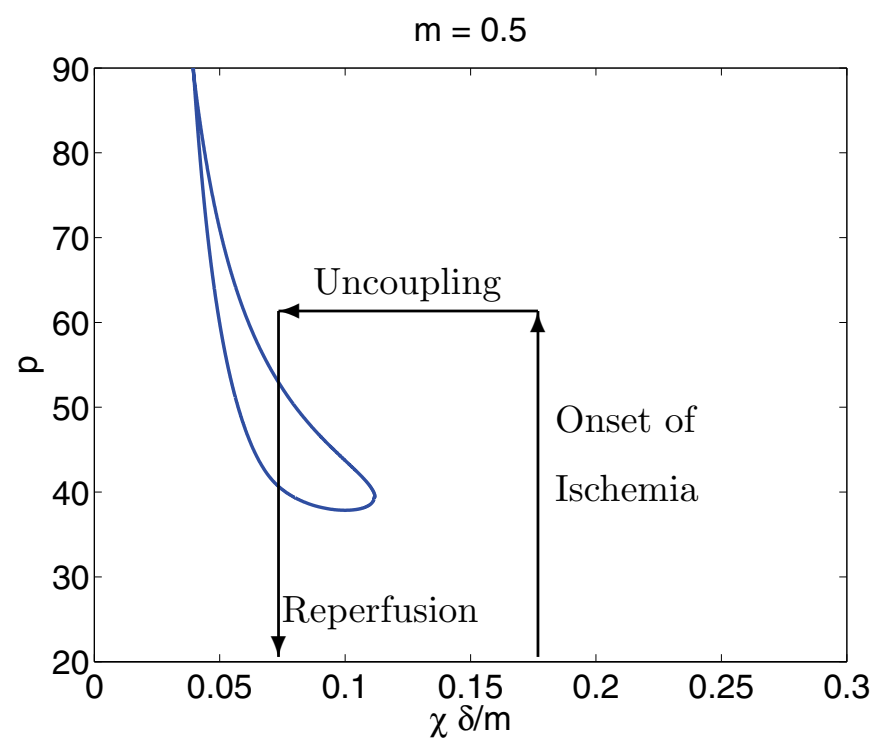

Figure 24. Coupled same-size LRI cells. Ischemic onset leading to gap junction uncoupling followed by reperfusion. The reperfusion trajectory intersects the region of oscillatory solutions generating a reperfusion arrhythmia.

rise. At this point in time, gap junctions begin uncoupling, causing a decrease in coupling strength. Upon reperfusion the degree of ischemia returns toward its nonischemic initial state but at a different coupling strength. If the uncoupling is sufficient, the trajectory of return to normalcy may intersect the oscillatory parameter region. This scenario illustrates a possible mechanism for reperfusion arrhythmias. Further experimentation is needed to corroborate this reperfusion arrhythmia mechanism.

6. Conclusion. A simple parametric change to ionic models recreates several features of ischemic tissue such as elevated transmembrane potential and shorter action potential duration. We couple an ischemic cell with this type of ischemic modification to a normal cell in two separate systems of ionics. We show that with both models there is a region in parameter space in which the normal cell oscillates. Furthermore, there are parameter regions in which coupled ischemic and normal cells oscillate, but when these cells are uncoupled, they are individually nonoscillatory. The ischemic cell while uncoupled maintains an elevated, stable, inexcitable resting potential. The normal cell is stable and excitable. We compare the coupled cell results in order to experiment and show that the models may assist in locating previously unobserved oscillatory behavior.

When normal cells are coupled to even small ischemic cells there is potential for automaticity. If an ischemic cell is large relative to a normal cell, there is little feedback from the normal cell to the ischemic cell. The normal cell reacts as a forced single cell subject to an applied constant current and a leak current. This observation leads to the small $m$ limit theory. This theory is for general ionic forms though the two sets of ionics we study here exemplify the theory. An interesting observation is that for the LRI model, the oscillations were calcium-induced and had no sodium current component. The explanation for this is found 
in the window currents for each of these ions. Modeling a drug-induced augmentation of the calcium window current suggested by experiments leads to the prediction that the parameter location of oscillatory behavior in the drug experiment is at higher coupling and lower ischemic levels than the drug-free experiment.

A mechanism for reperfusion arrhythmias is established based on the difference in time scales of elevation of extracellular potassium and uncoupling of gap junctions. Further experimentation will be necessary to confirm this reperfusion arrhythmia mechanism.

\section{Appendix A. RHH parameters.}

Table of values for the RHH model

\begin{tabular}{|c|c|c|}
\hline Symbol & Function & Value (units) \\
\hline $\mathrm{R}$ & Ideal gas constant & $8.315 \mathrm{~J} \mathrm{~mol}^{-1} \cdot \mathrm{K}^{-1}$ \\
\hline $\mathrm{T}$ & Temperature & $300 \mathrm{~K}$ \\
\hline $\mathrm{F}$ & Faraday's constant & $96.49 \times 10^{3} \mathrm{C} \cdot \mathrm{mol}^{-1}$ \\
\hline $\bar{g}_{N a}$ & Max sodium channel conductance & 120 (mho) \\
\hline $\bar{g}_{K}$ & Max potassium channel conductance & 36 (mho) \\
\hline$g_{L}$ & Leak channel conductance & 0.3 (mho) \\
\hline$V_{N a}$ & Sodium Nernst potential & $56(\mathrm{mV})$ \\
\hline$V_{L}$ & Leak (composite) Nernst potential & $-54.4(\mathrm{mV})$ \\
\hline$V_{K}$ & Potassium Nernst potential & $\frac{R T}{F} \ln \left(\frac{p}{K_{i}}\right)(\mathrm{mV})$ \\
\hline$K_{i}$ & Intercellular potassium concentration & $397(\mathrm{mM})$ \\
\hline$m_{\infty}(V)$ & $\begin{array}{l}\text { Steady state open probability for the } \\
\text { sodium channel activation gate }\end{array}$ & $\frac{\alpha_{m}(V)}{\alpha_{m}(V)+\beta_{m}(V)}$ \\
\hline$\alpha_{m}(V)$ & $\begin{array}{l}\text { Rate of sodium channel activation gate } \\
\text { opening }\end{array}$ & $0.1(\mathrm{~V}+40) /(1-\exp (-(\mathrm{V}+40) / 10))$ \\
\hline$\beta_{m}(V)$ & $\begin{array}{l}\text { Rate of sodium channel activation gate } \\
\text { closing }\end{array}$ & $4 \exp (-(\mathrm{V}+65) / 18)$ \\
\hline$n_{\infty}(V)$ & $\begin{array}{l}\text { Steady state open probability for the } \\
\text { potassium channel activation gate }\end{array}$ & $\frac{\alpha_{n}(V)}{\alpha_{n}(V)+\beta_{n}(V)}$ \\
\hline$\tau_{n}(V)$ & $\begin{array}{l}\text { Time constant for the potassium channel } \\
\text { activation gate dynamics }\end{array}$ & $\frac{1}{\alpha_{m}(V)+\beta_{m}(V)}$ \\
\hline$\alpha_{n}(V)$ & $\begin{array}{l}\text { Rate of potassium channel activation gate } \\
\text { opening }\end{array}$ & $0.01(\mathrm{~V}+55) /(1-\exp (-(\mathrm{V}+55) / 10))$ \\
\hline$\beta_{n}(V)$ & $\begin{array}{l}\text { Rate of potassium channel activation gate } \\
\text { closing }\end{array}$ & $0.125 \exp (-(\mathrm{V}+65) / 80)$ \\
\hline
\end{tabular}


Appendix B. LRI parameters. The equations and parameters for the LRI model.

\begin{tabular}{lll}
\hline Parameter name & Symbol & Value \\
\hline Intracellular potassium & $K_{i}$ & $145 \mathrm{mM}$ \\
Extracellular sodium & $N a_{o}$ & $140 \mathrm{mM}$ \\
Intracellular sodium & $N a_{i}$ & $18 \mathrm{mM}$ \\
Na/K permeability ratio & $P R_{N a K}$ & 0.01833 \\
Ideal gas constant & $\mathrm{R}$ & $8.315 \mathrm{~J} \mathrm{~mol}$ \\
Temperature & $\mathrm{T}$ & $310 \mathrm{~K}$ \\
Faraday's constant & $\mathrm{F}$ & $96.49 \times 10^{3} \mathrm{C} \cdot \mathrm{mol}^{-1}$ \\
\hline
\end{tabular}

\begin{tabular}{ll}
\hline Variable name & Symbol \\
\hline Transmembrane potential & $V$ \\
Sodium activation gating variable & $m$ \\
Sodium fast inactivation gating variable & $h$ \\
Sodium slow inactivation gating variable & $j$ \\
Calcium activation gating variable & $d$ \\
Calcium inactivation gating variable & $f$ \\
Potassium activation gating variable & $x$ \\
Intracellular calcium & $C a_{i}$ \\
\hline
\end{tabular}

\begin{tabular}{lll}
\hline Nernst potential & Symbol & Value \\
\hline Sodium & $V_{\mathrm{Na}}$ & $54.4 \mathrm{mV}$ \\
Calcium & $V_{\mathrm{Si}}$ & $7.77-13.0287 \log \left(C a_{i}\right)$ \\
Potassium & $V_{\mathrm{K}}$ & $\frac{R T}{F} \log \left(\left(K_{o}+P R_{N a K} N a_{o}\right) /\left(K_{i}+P R_{N a K} N a_{i}\right)\right)$ \\
Potassium & $V_{\mathrm{K} 1}$ & $\frac{R T}{F} \log \left(K_{o} / K_{i}\right)$ \\
Potassium & $V_{\mathrm{Kp}}$ & $V_{\mathrm{K} 1}$ \\
Background & $V_{b}$ & -59.87 \\
\hline
\end{tabular}


Probabilities for gating variables

For $V<-40$

$$
\begin{aligned}
a_{h}= & 0.135 \exp ((80+V) /(-6.8)) \\
a_{j}= & \left(-1.2714 \times 10^{5} \exp (0.2444 V)\right. \\
& \left.-3.474 \times 10^{-5} \exp (-0.04391 V)\right) \\
& \cdot(V+37.78) /(1+\exp (0.311(V+79.23))) \\
b_{h}= & 3.56 \exp (0.079 V)+3.1 \times 10^{5} \exp (0.35 V) \\
b_{j}= & 0.1212 \exp (-0.01052 V) /(1+\exp (-0.1378(V+40.14)))
\end{aligned}
$$

For $\mathrm{V} \geq 40$

$$
\begin{aligned}
& a_{h}=0 \\
& a_{j}=0 \\
& b_{h}=1 /(0.13(1+\exp ((V+10.66) /(-11.1)))) \\
& b_{j}=0.3 \exp \left(-2.535 \times 10^{-7} V\right) /(1+\exp (-0.1(V+32)))
\end{aligned}
$$

For all $V$

$$
\begin{aligned}
& a_{m}=0.32(V+47.13) /(1-\exp (-0.1(V+47.13))) \\
& b_{m}=0.08 \exp (-V / 11) \\
& a_{d}=0.095 \exp (-0.01(V-5)) /(1+\exp (-0.072(V-5))) \\
& b_{d}=0.07 \exp (-0.017(V+44)) /(1+\exp (0.05(V+44))) \\
& a_{f}=0.012 \exp (-0.008(V+28)) /(1+\exp (0.15(V+28))) \\
& b_{f}=0.0065 \exp (-0.02(V+30)) /(1+\exp (-0.2(V+30)))
\end{aligned}
$$

For $V>-100$

$$
\begin{array}{r}
x_{i}=2.837(\exp (0.04(V+77))-1) / \\
((V+77) \exp (0.04(V+35)))
\end{array}
$$

For $V \leq-100$

$$
\begin{aligned}
x_{i}=1 & \\
a_{x}= & 0.0005 \exp (0.083(V+50)) /(1+\exp (0.057(V+50))) \\
b_{x}= & 0.0013 \exp (-0.06(V+20)) /(1+\exp (-0.04(V+20))) \\
a_{k 1}= & 1.02 /\left(1+\exp \left(0.2385\left(V-V_{K 1}-59.215\right)\right)\right) \\
b_{k 1}= & \left(0.49124 \exp \left(0.08032\left(V-V_{K 1}+5.476\right)\right)\right. \\
& \left.\quad+\exp \left(0.06175\left(V-V_{K 1}-594.31\right)\right)\right) / \\
& \left(1+\exp \left(-0.5143\left(V-V_{K 1}+4.753\right)\right)\right)
\end{aligned}
$$

$K p$ conductance $\quad K_{p}=1 /(1+\exp ((7.488-V) / 5.98))$

\begin{tabular}{lll}
\cline { 1 - 2 } Channel conductance & Symbol and value & \\
\cline { 1 - 2 } Sodium & $g_{N a}=23 m^{3} h j$ & \\
Calcium & $g_{S i}=0.09 d f$ & Membrane capacitance \\
Potassium & $g_{K}=0.282 \sqrt{K_{o} / 5.4} x x_{i}$ & $C_{m}=1$ \\
Potassium & $g_{K 1}=0.6047 \sqrt{K_{o} / 5.4} \frac{a_{k 1}}{a_{k 1}+b_{k 1}}$ & \\
Potassium & $g_{K p}=0.0183 K_{p}$ & \\
Background & $g_{b}=0.03921$ &
\end{tabular}




\begin{tabular}{ll}
\hline Currents & Symbol and value \\
\hline Sodium & $I_{N a}=g_{N a}\left(V-V_{N a}\right)$ \\
Calcium & $I_{S i}=g_{S i}\left(V-V_{S i}\right)$ \\
Potassium & $I_{K}=g_{K}\left(V-V_{K}\right)$ \\
Potassium & $I_{K 1}=g_{K 1}\left(V-V_{K 1}\right)$ \\
Potassium & $I_{K p}=g_{K p}\left(V-V_{K p}\right)$ \\
Background & $I_{b}=g_{b}\left(V-V_{b}\right)$ \\
\hline
\end{tabular}

$$
\begin{aligned}
& \text { Equations } \\
& \qquad \begin{aligned}
\frac{d V}{d t} & =-1 / C_{m}\left(I_{N a}+I_{S i}+I_{K}+I_{K 1}+I_{K p}+I_{b}\right) \\
\frac{d y}{d t} & =\frac{y_{\infty}-y}{\tau_{y}} \\
\frac{d C a_{i}}{d t} & =-10^{-4} I_{S i}+0.07\left(10^{-4}-C a_{i}\right)
\end{aligned}
\end{aligned}
$$

$$
\text { for } y \in\{m, h, j, d, f, x\} \text { and } y_{\infty}=a_{y} /\left(a_{y}+b_{y}\right), \tau_{y}=1 /\left(a_{y}+b_{y}\right) .
$$

\section{Appendix C. Small $m$ limit theorem.}

Theorem C.1. Let $L^{1}\left(T^{n}\right)$ be the space of periodic functions on $R^{n}$ of period $T$ with bounded $L^{1}$ norm on $[0, T]$. Let $x, y \in L^{1}\left(T^{n}\right)$ and $H, G: L^{1}\left(T^{n}\right) \rightarrow L^{1}\left(T^{n}\right)$ be $C^{1}$ maps. Let ${ }^{\prime} \equiv \frac{d}{d t}$.

Assumption A3. Suppose

$$
y^{\prime}-H(y)=I-D_{1} y
$$

has a periodic solution, $Y(t)$, for some constant matrix $D_{1} \in R^{n \times n}$ and constant vector $I \in R^{n}$. Assumption A4. Suppose

$$
x^{\prime}-G(x)=0
$$

has the steady state solution, $x^{*}$, such that $I=D_{1} x^{*}$.

Then the coupled system

$$
\begin{aligned}
& m_{y}\left(y^{\prime}-H(y)\right)=\delta_{1}(x-y), \\
& m_{x}\left(x^{\prime}-G(x)\right)=\delta_{2}(y-x)
\end{aligned}
$$

has a periodic solution for $\frac{m_{y}}{m_{x}}$ sufficiently small with $m_{y}$ and $m_{x}$ scalars and matrices $\delta_{i}=$ $m_{y} D_{i}$ for $i=1,2$.

Proof outline. We look for periodic solutions $(y, x) \in L^{1}\left(T^{n}\right) \times L^{1}\left(T^{n}\right)$ that are extended from the periodic solution $(Y(t), 0)$ when $\epsilon=\frac{m_{y}}{m_{x}}=0$ to $\epsilon>0$. To do this we transform our nonlinear problem using the Lyapunov-Schmidt method into an invertible linear problem plus higher order terms. We then invoke the implicit function theorem which yields a unique perturbed solution $\left(Y+h_{1}(\epsilon), h_{2}(\epsilon)\right)$ for sufficiently small $\epsilon$. For details see [16].

Now we relate Theorem C.1 to the coupled system (2.3)-(2.6). Let $y=\left(V_{1}, \mathbf{w}_{1}\right)^{T}$ and $x=\left(V_{2}, \mathbf{w}_{2}\right)^{T}$ with $H(y)=\left(F\left(y, \mathbf{p}_{0}\right), g(y)\right)^{T}$ and $G(x)=(F(x, \mathbf{p}), g(x))^{T}$. Also let $D_{1}$, $D_{2}$, and $M$ each be such that $D_{1}^{1,1}=d=\frac{\chi \delta}{m}, D_{1}^{i, j}=0$ otherwise, $D_{2}^{1,1}=\frac{\chi \delta}{1-m}, D_{2}^{i, j}=0$ otherwise, and $M_{1,1}=1, M_{i, j}=0$ otherwise. With this choice, there is coupling only in the transmembrane potential equation.

\section{REFERENCES}

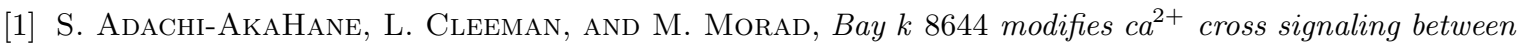
dhp and ryanodine receptors in rat ventricular myocytes, Amer. J. Physiology, 276 (1999), pp. H1178H1189. 
[2] M. A. Beardslee, D. L. Lerner, P. N. Tadros, J. G. Laing, E. C. Beyer, K. A. Yamada, A. G. KlÉBer, R. B. Schuessler, AND J. E. SAfFitz, Dephosphorylation and intracellular redistribution of ventricular connexin 43 during electrical uncoupling induced by ischemia, Circulation Research, 87 (2000), pp. 656-662.

[3] E. CARmeliet, Cardiac ionic currents and acute ischemia: From channels to arrhythmias, Physiological Reviews, 79 (1999), pp. 917-1017.

[4] W. E. Cascio, T. A. Johnson, And L. S. Gettes, Electrophysiologic changes in ischemic ventricular myocardium: I. Influence of ionic, metabolic, and energetic changes, J. Cardiovasc. Electrophysiol., 6 (1995), pp. 1039-1062.

[5] F. F. T. Ch'en, R. D. Vaughan-Jones, K. Clarke, and D. Noble, Modelling myocardial ischemia and reperfusion, Progress in Biophysics and Molecular Biology, 69 (1998), pp. 515-538.

[6] P. DAleau, Lysophosphatidylcholine, a metabolite which accumulates early in myocardium during ischemia, reduces gap junctional coupling in cardiac cells, J. Mol. Cell Cardiol., 31 (1999), pp. 13911401.

[7] E. J. Doedel, A. R. Champneys, T. F. Fairgrieve, Y. A. Kuznetsov, B. Sandstede, And X. WANG, AUTO97: Continuation and Bifurcation Software for Ordinary Differential Equations, Concordia University, Montreal, Canada, 1997.

[8] R. FitzHugh, Thresholds and plateaus in the Hodgkin-Huxley nerve equations, J. Gen. Physiol., 43 (1960), p. 867.

[9] R. FitzHugh, Impulses and physiological states in theoretical models of nerve membrane, Biophys. J., 1 (1961), pp. 445-466.

[10] A. S. HARris AND A. G. Rojas, The initiation of vantricular fibrillation due to coronary occlusion, Exp. Med. Surg., 1 (1943), pp. 105-122.

[11] M. J. JANSE AND A. L. WIT, Electrophysiological mechanisms of ventricular arrhythmias resulting from myocardial ischemia and infarction, Physiological Reviews, 69 (1989), pp. 1049-1169.

[12] J. Keener And J. Sneyd, Mathematical Physiology, Interdiscip. Appl. Math. 8, Springer-Verlag, New York, 1998.

[13] R. Kumar And R. W. Joyner, An experimental model of the production of early after depolarizations by injury current from an ischemic region, Pflugers Arch., 428 (1994), pp. 425-432.

[14] R. Kumar, R. Wilders, R. W. Joyner, H. J. Jongsma, E. Verheijck, D. A. Golod, A. C. G. VAN GinneKen, AND W. N. GoOLSBy, Experimental model for an ectopic focus coupled to ventricular cells, Circulation, 94 (1996), pp. 833-841.

[15] C.-H. LuO AND Y. Rudy, A model of the ventricular cardiac action potential; depolarization repolarization and their interaction, Circulation Research, 68 (1991), pp. 1501-1526.

[16] B. E. Peercy, Models of Border Zone Arrhythmias in Acute Myocardial Ischemia, Ph.D. thesis, University of Utah, Salt Lake City, UT, 2003.

[17] N. S. Peters, C. R. Green, P. A. Poole-Wilson, and N. J. Severs, Reduced content of connexin 43 gap junctions in ventricular myocardium from hypertrophied and ischemic human hearts, Circulation, 88 (1993), pp. 864-875.

[18] S. Picard, R. Rouet, P. Ducouret, P. E. Puddu, F. Flais, A. Criniti, F. Monti, And J.-L. GÉRARD, $K_{a t p}$ channels and 'border zone' arrhythmias: Role of the repolarization dispersion between normal and ischaemic ventricular regions, British J. Pharmacology, 127 (1999), pp. 1687-1695.

[19] R. Rouet, M. Adamantidis, E. Honore, And B. Dupuis, In vitro abnormal repetitive responses in guinea pig ventricular myocardium exposed to combined hypoxia, hyperkalemia and acidosis, J. Appl. Cardiol., 4 (1989), pp. 19-29.

[20] R. Rouet, S. Picard, C. Libersa, M. Ghadanfar, C. Alabaster, and J.-L. Gérard, Electrophysiological effects of dofetilide in an in vitro model of "border zone" between normal and ischemic/ reperfused myocardium, Circulation, 101 (2000), pp. 86-93.

[21] R. C. TAN AND R. W. JOYNER, Electrotonic influences on action potentials from isolated ventricular cells, Circulation Research, 67 (1990), pp. 1071-1081.

[22] R. C. TAN, T. Osaka, AND R. W. Joyner, Experimental model of effects on normal tissue of injury current from ischemic region, Circulation Research, 69 (1991), pp. 965-974.

[23] K. A. Yamada, J. McHowat, G.-X. Yan, K. Donahue, J. Peirick, A. G. Kléber, And P. B. Corr, Cellular uncoupling induced by accumulation of long-chain acylcarnitine during ischemia, Circulation Research, 74 (1994), pp. 83-95. 